\title{
Design and Development of Y81-1000 Type Heavy Hydraulic Scrap Baling Press
}

\author{
Mingbo LI ${ }^{1,2}$, Zhongming CHEN, Yihua SUN ${ }^{1, *}$, Gao LIN², Yijun TAO², \\ Shuiping $\mathrm{LI}^{3}$, Zhengrong $\mathrm{LIU}^{2}$, Min YOU ${ }^{1}$
}

${ }^{1}$ School of materials and chemical engineering, China Three Gorges University, Yichang, 443002, China

${ }^{2}$ Hubei Lidi Machine Tool Co.,Ltd, Yichang, 443001, China,

${ }^{3}$ School of mechanical and electronic engineering, Wuhan University of Technology, Wuhan, 430070, China

email: sunny.hust@ctgu.edu.cn

\begin{abstract}
Keywords: Scrap metal processing; Heavy packing machine; Development; Resource Recovery Abstract. To summarize the main technical features of the international heavy hydraulic scrap baling press, the technical characteristics of Y81-1000 type heavy oil-hydraulic scrap baling press made in China were mainly introduced. At the same time, the new design and development of this technical equipment were introduced in detail from technical background to integral structure design, from electric control system to hydraulic system in this paper.
\end{abstract}

\section{Introduction}

Nowadays, the development of economy and society is facing the exhaustion of primary resources and the crisis of traditional energy. The green economy and circular economy have become a new trend of global sustainable development [1]. As a kind of important energy saving and emission reduction and renewable resources, scrap iron and steel have received great attention [2-4]. It has very important practical significance that the scrap iron and steel resources had been effectively exploited and utilized in order to save resources and protect the environment. At present, the scrap ratio in the iron and steel industry of developed countries had increased to more than 40-50\% [3,4]. In China, by contrast, it only maintained at a very low level of $14-23 \%$. So the utilization of steel scrap had become a long-term strategic policy for the reform and development of the iron and steel industry in China [3,4]. The scientific classification processing of steel scrap and the scrap concentrate steelmaking which have yet to be resolved had become an important issue in metallurgical industry.

In recent years, although the sort and quantity of scrap steel processing equipment are increasing, the requirement of social development has not been satisfied in China. The backward equipment in the iron and steel scrap processing had stood in the way of the iron and steel production enterprise stepping forward. It is a vital factor also that the increase of scrap ratio was restricted in the iron and steel industry [4]. Meanwhile, with the development of economic modernization, kinds of scrap steel became more and more, such as large structures, containers, waste planking and so on, which is difficult to process. So larger processing capacity and range of working parameters were proposed to be used in the scrap processing equipment [4]. In developed country, oil-hydraulic scrap baling press is developing towards large scale which has reached more than 1500 tons for processing capacity. Therefore, it is one of the effective ways that the sustainable development in iron and steel industry will be promoted by the development of large-scale, automated, high-efficiency scrap processing technology and equipment.

All in all, both processing method of steel scrap and the mechanical equipment for processing steel scrap are not as advanced as that in developed countries [3]. The steel scrap in China is still mainly processed by manpower and simple mechanical device. Moreover, additional encouragement for investment in scrap steel industry would be beneficial[3]. The needs for heavy oil-hydraulic scrap baling press had existed in domestic market, combining with the actual situation in China, the design and development and serialization of heavy oil-hydraulic scrap baling press 
seemed particularly urgent. In this paper, a Y81-1000 type oil-hydraulic scrap baling press is introduced in detail from technical background to integral structure design, from electric control system to hydraulic system, and the like.

\section{Technical background}

The manufacturer of heavy oil-hydraulic scrap baling press is a few internationally, Germany Lindeman company, Italian Vezzani company and America Harris corporation are the typical manufacturer for heavy oil-hydraulic scrap baling press. 1000t oil-hydraulic scrap baling press all lies their production inventory. The main technical features of this type production made by different manufacturers are compared and analyzed as follows:

The main technical features of the production of Lindeman company: (1) Three direction extrusion, (2) Shear mechanism equipped ( as the first stage pressure head go ahead, the waste material stretched out container is cut off ), (3)The ejector door along the vertical direction.

The main technical features of the production of Vezzani company: (1) Three direction extrusion, (2) The block rack fixed on three sides of the container, (3) The ejector door along the horizontal direction in the lateral.

The main technical features of the production of Harris corporation: (1) Three direction extrusion; (2) The feeding hopper fixed on one side of the container, on the other side the prepressing cover is equipped.(3)The ejector door along the horizontal direction in the lateral.

It can be found that the three direction extrusion is all used to product high density and high yield of scrap bales. However, there lies different in the continuous feed processing and design of the ejector door. After hopper installed continuous feeding can be realized by Lin Deman's production. But because of no pre-extrusion cover, the processing capacity for large structures is limited. The ejector door along the vertical direction makes the system compact. Vizani's production can achieve continuous feeding, but the structure is not compact enough. Harris' production possesses the processing capacity for large structures, but continuous feeding can not be achieved due to the main compactor without longer carriage.
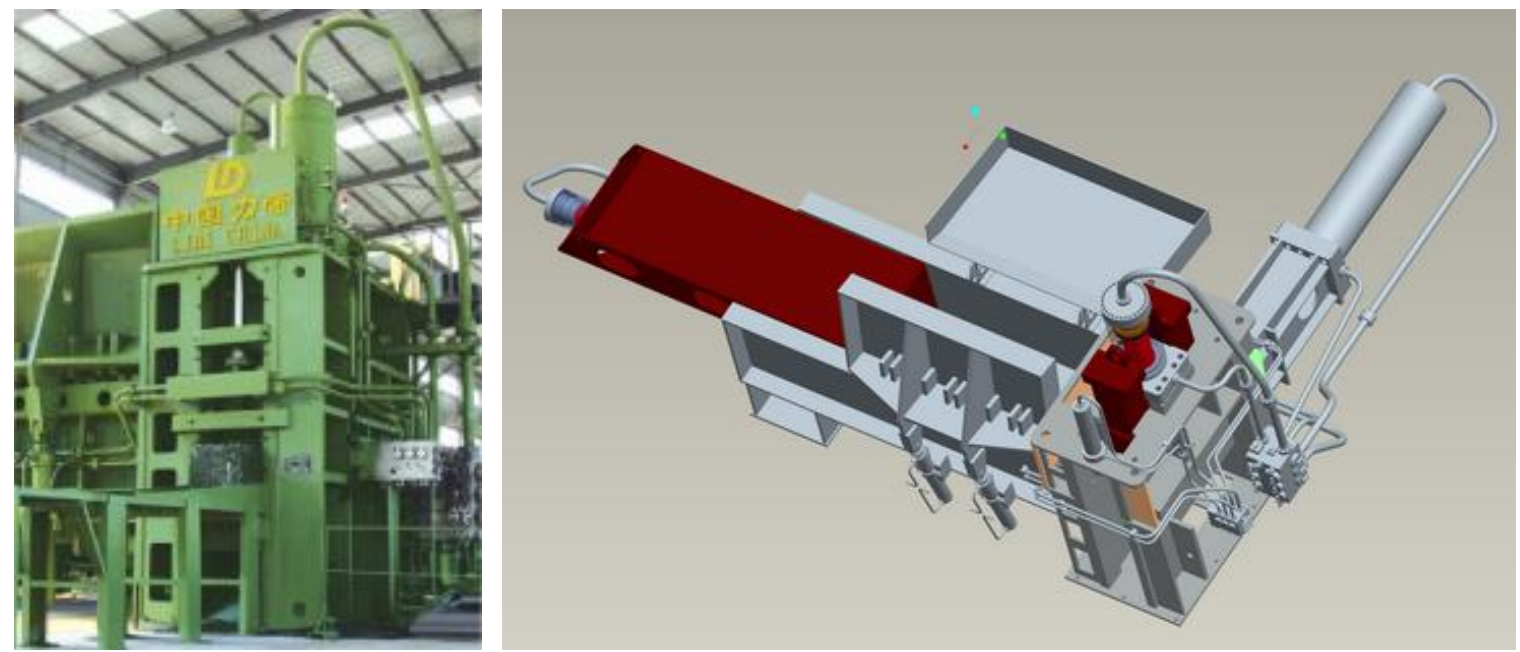

Fig.1. The machine in production (left) and its three-dimensional diagrams (right)

After the aforementioned comparison and analysis, some technical requirements were used in Y81-1000 type heavy oil-hydraulic scrap baling press, such as lateral pre-extrusion cover, main compactor with longer carriage, ejector door along vertical direction and other automation equipment, as shown in Fig. 1. Although the structure are slightly complicated and the cost is higher, the advantages of wide applicability, high productivity and automation, security enhancement, and prolong service longevity make it a good prospect of market. 


\section{Creativity and advancement of Y81-1000 type machine}

Integral structure design. Triaxial compression method (i.e. X, Y, Z direction) was used to achieve high density scrap block $\left(2.2-3.2 \mathrm{~g} / \mathrm{cm}^{3}\right)$ which can effectively cut down the storage area and improve the transporting efficiency and reduce the heat loss of smelting process [5], as shown in Fig. 1.

Structure of spacious door frame without restriction was designed for pushing out bales unrestrictedly which result in reducing the friction loss and prolonging the service life of wearing plates in high pressure chamber.

Taking a long compactor carriage with scraper and stopper device designed [6], it can make the machine on the next feeding operation while bales pressing operation. Therefore, the machine can continuous and efficient production [7].

The main part of the machine is of frame welding structure using super thick steel, strong and durable. Ultra large feeding box processes adaptability for steel scrap to some extent and a lot of HARDOX plate are used as wearing plates. The lateral extrusion press cover and feed hopper equipped make it without waiting for loading time as processing a large size of scrap steel structure [8-10].

Electric control system. According to system demands, it is chosen and designed for hardware and software of the control system, and SIMATIC S7-300 programmable controller of SIEMENS Corporation is chosen. By taking S7-300 PLC as main unit, the programme for machine combining with safety protection program was redeveloped.

The electric control system of this machine is made up of the Programmable Controller (PLC) and the man-machine interface monitoring system and network remote diagnosis monitoring system. Siemens S7-300 program controller is to achieve PLC logic control, as seen in Fig. 2, with the switch input and output module and the multi-channel analog module included. The non-contact proximity switch and magnetostrictive sensor are used to monitor the valve status and displacement respectively to make the oil level, oil temperature and hydraulic pressure accurately controlled [11].

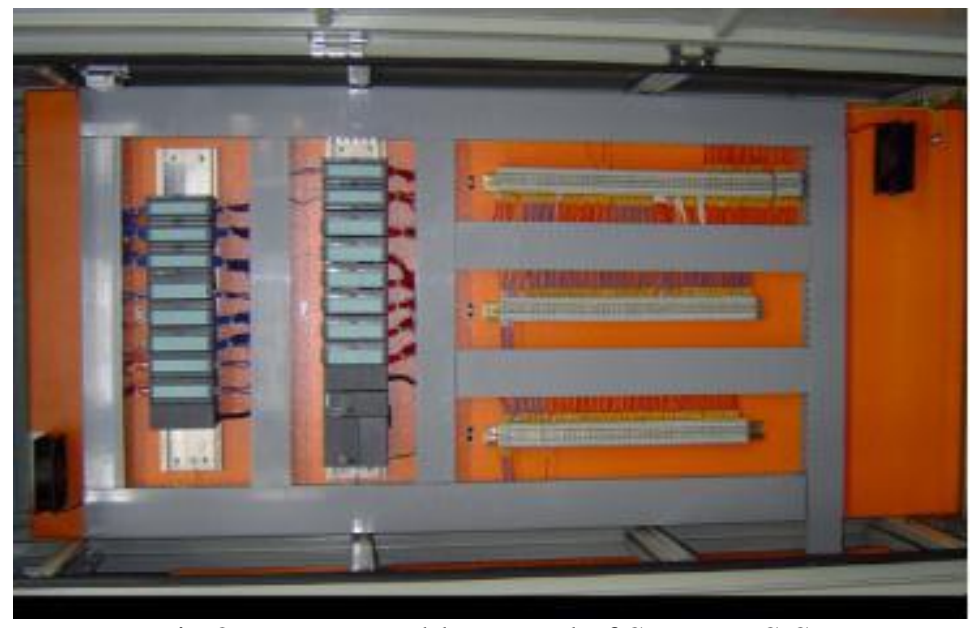

Fig.2. Programmable control of SIEMENS S7

The information of operating status or fault messages can be shown in real time on the man-machine interface display in the control system, such as cylinder position and pressure, solenoid valve and oil valve switch state, oil level and temperature and so on. All user parameters can be adjusted.

Equipped with network data transmission and monitoring module, the various status signals can be transmitted to the remote terminal through Internet, so the remote diagnosis and treatment of equipment operating status can be achieved.

Hydraulic system. The integrated valve block and cartridge valves are adopted in the hydraulic system to make it advantage in compact structure and progress greatly in the reliability of operation. As shown in Fig. 3, the large integrated valve block is a key component of the whole hydraulic system with a high technology content which was imported originally. Now through cooperative 
researched and developed with the domestic professional manufacturers, it has been successfully developed which had possessed reliable performance and independent intellectual property rights.

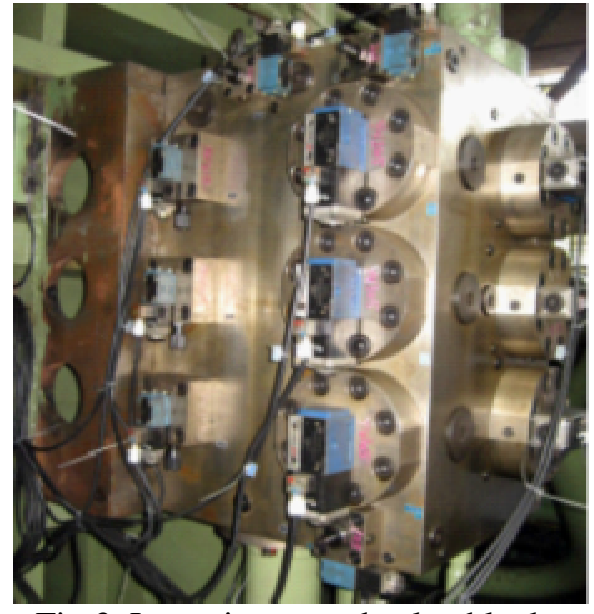

Fig.3. Large integrated valve block

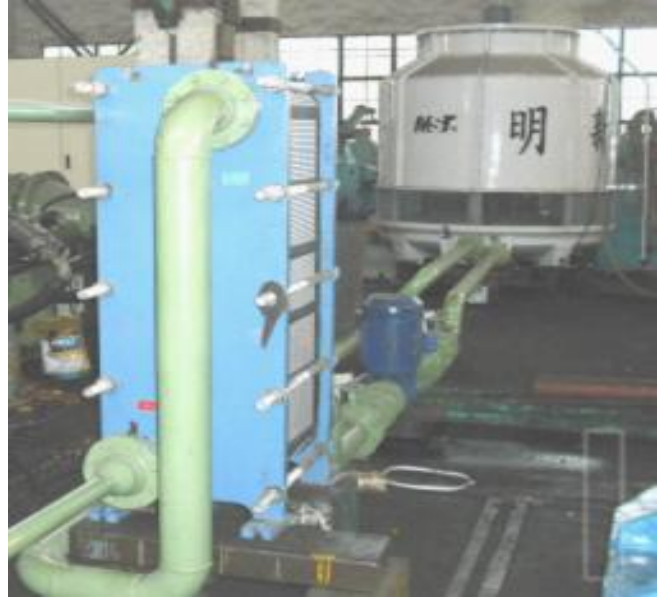

Fig.4. Water cooling tower, pump and heat exchanger

Hydraulic principle adopts a combination logic distribution mode of low pressure with high flow and high pressure with small flow. At the early and middle stage of heavy oil-hydraulic scrap baling press, a low pressure and high flow is needed. At the last stage, high pressure and small flow is a reasonable operational principle. As the logic mode adopted the installed power is reduced significantly and the energy efficiency is enhanced in this machine.

A automatic circulating water cooling device was designed and developed to control the heat accumulation in hydraulic system which include a pipeline pump, a heat exchanger and a cooling tower, as shown in Fig. 4. Compared with the traditional techniques, the cooling effect is better and the cooling water is only recycled in the system which reduced the waste of water and the cost.

Table 1 the key technical indexes of this machine and Lindeman product

\begin{tabular}{l|l|l}
\hline \multicolumn{1}{c|}{ Index name } & This machine & Lindeman \\
\hline Main pressure $(\mathrm{kN})$ & 10000 & 10000 \\
\hline Secondary pressure $(\mathrm{kN})$ & 5000 & 5000 \\
\hline Feed box size $(\mathrm{mm})$ & $5510 \times 2000 \times 1500$ & $5500 \times 2000 \times 1600$ \\
\hline Nominal pressure $(\mathrm{MPa})$ & 31.5 & 31.5 \\
\hline Cycle time $(\mathrm{Sec})$ & 95 & 98 \\
\hline Bale size $(\mathrm{mm})$ & $800-1500 \times 600 \times 600$ & $800-1500 \times 600 \times 600$ \\
\hline Bale density $\left(\mathrm{kg} / \mathrm{m}^{3}\right)$ & $2200-3200$ & $2000-3300$ \\
\hline No. of bales $(\mathrm{bales} / \mathrm{h})$ & $38-40$ & $38-40$ \\
\hline Main motor power $(\mathrm{kW})$ & $5 \times 90$ & $5 \times 90$ \\
\hline Weight $(\mathrm{t})$ & 260 & 250 \\
\hline Dimension $(\mathrm{mm})$ & $25700 \times 16710 \times 7240$ & $30100 \times 16810 \times 7200$ \\
\hline
\end{tabular}

\section{Summary}

As seen in Table 1, compared with the similar products of German Linderman in key technical index, Y81-1000 type heavy oil-hydraulic scrap baling press have reached the advanced level of similar products in the world. It has been identified as independent innovation products in Hubei Province of China. The technical equipment cater to the domestic requirements of large-scale, automation, high efficiency scrap baling press and can substitute for imported products leading to reduce the investment of scrap processing enterprises. This technical equipment is conducive to the increase of the scrap ratio and steel quality in the metallurgical industry of China for the circular 
economy and sustainable development. It is great significance to the technological progress and industrial structure optimization and upgrading of renewable resources utilization industry in China.

\section{References}

[1] Junming Zhu, Marian R. Chertow: Environmental Science and Technology Vol. 50 (2016), p. 2175

[2] Derek L. Diener, Anne-Marie Tillman: Conservation and Recycling Vol. 110 (2016), p. 48

[3] Xu Tang, Shiyuan Hu, Yaolin Liu, Hong Geng: Resources, Conservation and Recycling Vol. 109 (2016), p. 78

[4] Yanni Xuan, Qiang Yue: Resources, Conservation and Recycling Vol. 109 (2016), p. 1

[5] Linsheng Qin, Mingbo Li, Menglin Li, Junxia Zhang. CN 101920578. (2010)

[6] Mingbo Li, Gao Lin, Jianguo Liu , Li Ma, Rui Li. CN 204869749 U. (2015)

[7] Mingbo Li, Gao Lin, Fanrong Luo. CN 204869770U. (2015)

[8] Mingbo Li, Gao Lin, Yunqi Feng. CN 202412766 U. (2012)

[9] Mingbo Li, Gao Lin, Fanrong Luo. CN 204160798U. (2015)

[10] Mingbo Li, Gao Lin, Fanrong Luo. CN 105459432A. (2016)

[11] Mingbo Li, Gao Lin, Denghuan Ni. CN 204869768U. (2015) 


\title{
Studies and Implementation of Data Interface based on Domino Application System
}

\author{
Zhihong Ruan ${ }^{1, a}$, JunFeng Zhang ${ }^{1, b}$, Yi Jin',c \\ 'School of Education Science, Guizhou Normal University, Guiyang, Guizhou, 550001, P. R. China \\ ${ }^{2}$ Department of Computer Science Guiyang University, Guiyang, Guizhou, 550001, P. R. China \\ asmall_russet@126.com, bgzsd2008@126.com, c16279138@qq.com
}

Keywords: Domino, Data interface, Application system, XML

Abstract. For solving the shortages of the existing methods of data exchange among the various enterprise information systems, in this paper, the XML documents were used as the carrier of structured data to design and implement a new type of data interface based on Donimo application system. By using FTP service to communicate, the loose coupling architecture was built to reduce the dependence and complexity of systems, and the security of the systems was improved. The new type of data interface is more efficient and more practical, and the environment of application systems will be also more efficient and more flexible by using it.

\section{Introduction}

At present in many enterprises the application systems based on Domino are used widely. Compared with other relational database systems, this kind of Domino-based application system uses text-type database.

During the process of data exchange and sharing among these databases, general methods are used basically in the following: Lotus domino's ODBC Interface, Lotus LEI tools and Lotus ESB interface. But the shortcomings of these conventional solutions such as the high cost, the platform choosing and unstructured data can not be handled and so on. Therefore, to analyzed and implemented the interface for solving these problems mentioned above is the focus of this paper.

\section{Principle of Interface Development}

\section{Principle of Text Type Database Generated XML}

There are two methods in Domino environment to process XML, one is using Java agent and the other one is using Lotus Script agent.

(1) For the method of Java Agent to generate the XML document. Because the built-in explanation of the XML capabilities of Domino Notes system, by bringing in the java packages, XML files can be generated through the DOM method. By using this way, there are no specific requirements for the operating system in which the application system run. As the method to generate xml file by using dom, so the special characters of some data fields can be explained automatically by the system.

(2) For the method of using Lotus script agent, XML files can be generated by lotus script invoking MSXML component, as the first method, the special characters of data fields of the XML files can be explained automatically by the system too, but there is one limitation for the operating system - it is effective only for the Windows operation system. Or the XML character string can be spliced directly by using lotus script, and then it can be written into the xml files.

\section{Interactive Data of Domino-based Application Systems}

The method of Domino application system to generate XML files mainly is used to help the Domino-based application systems generating interactive data which other application systems need. For the interactive data generated by other application systems, Domino-based application systems will be analyzed.

There are two methods for other application systems to generate interactive data; both of them can be explained as the following below: 
Method 1: Other application systems can invoke the application web agent of Domino by using the method of Http\&Get ; there are parameters which need to be delivered within URL. Then those parameters will be handled by the application web agent of Domino.

Method 2: XML files can be generated by other application systems, then can be delivered to servers which Domino application systems run in, and those XML files were read, analyzed by Domino application systems with timing java agent.

\section{Analysis and Generation Method of Other Application Systems}

The frameworks of other application systems basically divided into three types: (1) java and relational database (2) .NET and relational database (3) PHP and relational database.

For the systems structured by java, there are no specific requirements to operation systems, either Windows or Unix systems. XML files can be analyzed and handled by the following methods:

(1) DOM (Document Object Model) Method

DOM method is a collection of hierarchical organizational nodes or pieces of information. It is a cross-platform and language-independent convention for representing and interacting with objects in HTML, XHTML and XML documents. Aspects of the DOM may be addressed and manipulated within the syntax of the programming language in use. The public interface of a DOM is specified in its application programming interface (API), Figure 1 shows the method of DOM.

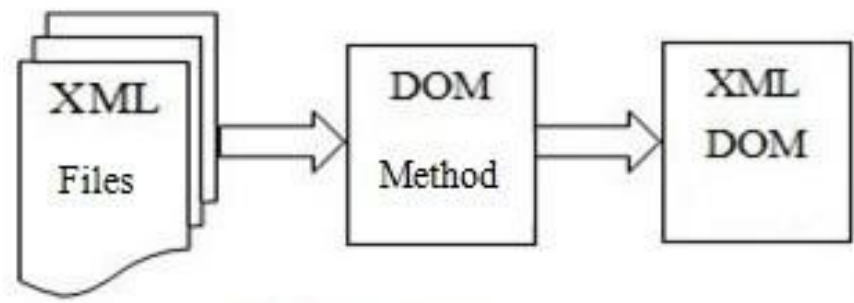

Fig. 1 DOMMethod

(2) SAX (Simple API for XML) Method

SAX (Simple API for XML) is a sequential access parser API for XML. SAX provides a mechanism for reading data from an XML document. It is a popular alternative to the Document Object Model (DOM).

SAX parsers have certain benefits over DOM-style parsers. The quantity of memory that a SAX parser must use in order to function is typically much smaller than that of a DOM parser. DOM parsers must have the entire tree in memory before any processing can begin, so the amount of memory used by a DOM parser depends entirely on the size of the input data. Because of the event-driven nature of SAX, processing documents can often be faster than DOM-style parsers.

Memory allocation takes time, so the larger memory footprint of the DOM is also a performance issue.Due to the nature of DOM, streamed reading from disk is impossible. Processing XML documents larger than main memory is also impossible with DOM parsers but can be done with SAX parsers. However, DOM parsers may make use of disk space as memory to sidestep this limitation. The method of SAX is shown in Figure 2.

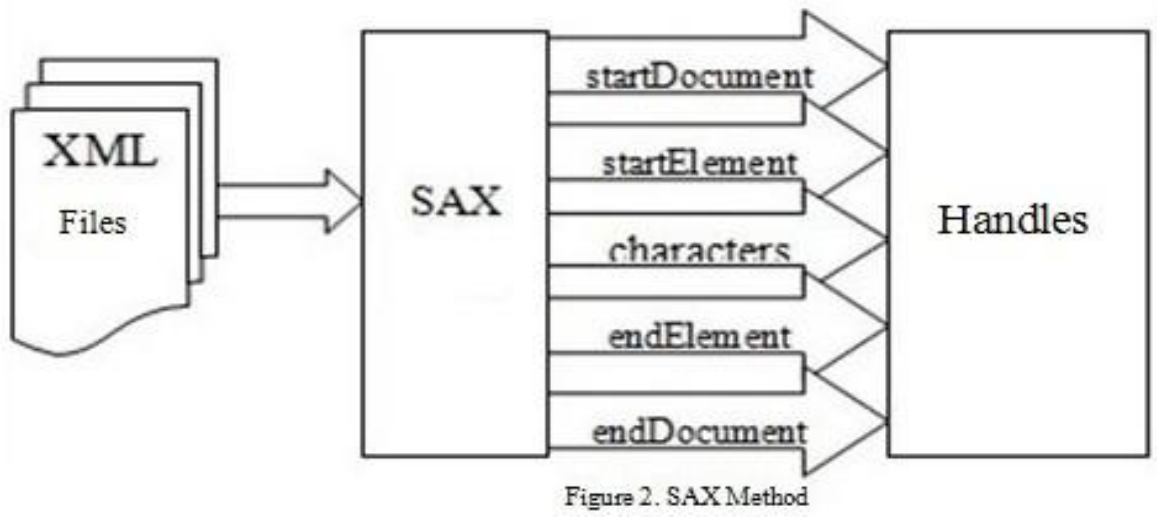

JDOM is an open source Java-based document object model for XML that was designed specifically for the Java platform so that it can take advantage of its language features. JDOM 
integrates with Document Object Model (DOM) and Simple API for XML (SAX), supports XPath and XSLT. It uses external parsers to build documents. JDOM was developed by Jason Hunter and Brett McLaughlin starting in March 2000. It has been part of the Java Community Process as JSR 102, though that effort has since been abandoned.

For the systems built by using .Net language, the method to analyze and process XML files is by using System.XML of .NET Framework. As .Net Framework is only compatible with Microsoft's operating system, so this method only can be used in the Microsoft windows operating system.

For the systems built by using PHP language, the method to analyze and process XML files is by using DOM and SimpleXML. Compared with .Net Framework, PHP platform has no special requirements on the operating system.

Based on what we have mentioned above all, one cross-platform interface based on Domino can analyze and process Xml files by using Domino java agent for avoiding the complexity of using lotus script agent. After interface data generated, timing FTP scripts were chosen in Domino-based application systems in java agent to achieve data transmission, and the agent program execution time was set, the time interval is 5 minutes. As a result, this interface can be deployed in both Unix system and Windows system, it provides cross-platform data transmission service.

\section{Implementation of Interactive Interface}

\section{Interactive Interface Description}

The data which other application systems need were generated as XML files according to the arranging XML format in the Domino-based application systems platforms, then these XML files were uploaded into the specified directory of other application systems by using FTP method.

Other application systems trigger the operation regularly several times a day to read the XML file automatically, obtained data services in accordance with the agreed XML format and provide the data synchronization in those database of other application systems. The interface data flow is shown in Figure 3 as below.

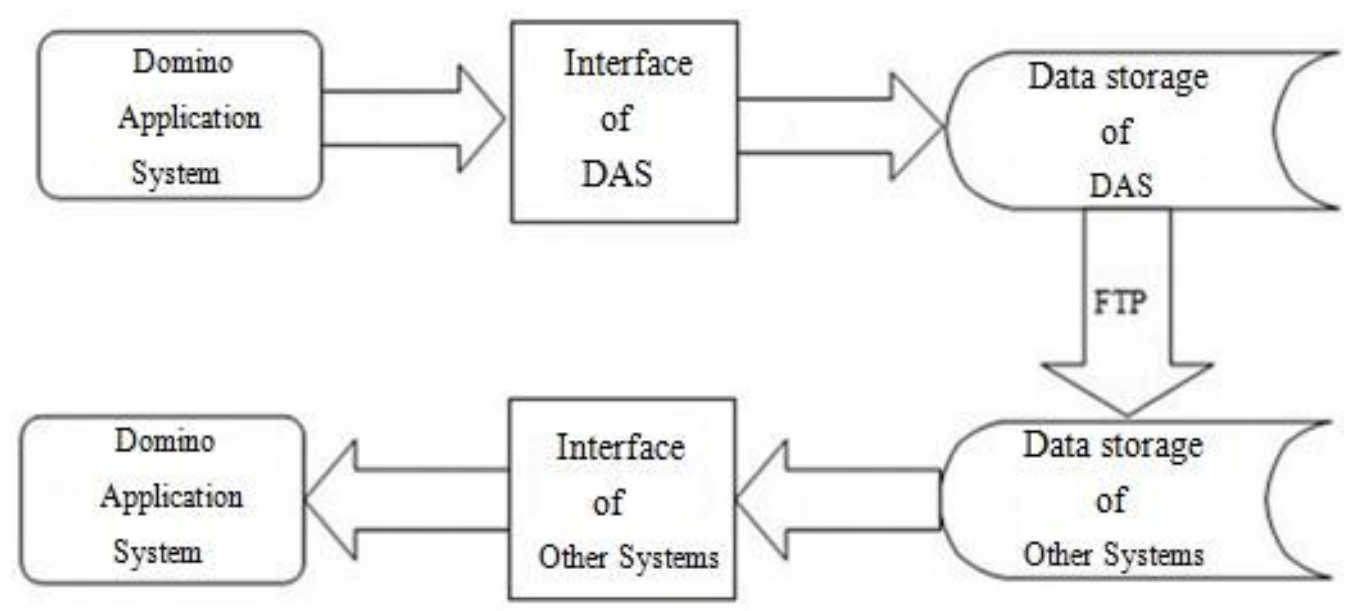

Figure 3. Data Flow of interative interiace

\section{Steps of Interface Development}

The following items are the steps of interface developing:

(1)Domino Database:

New database interfaces display database jkzs.nsf, specify the template is empty.

(2) Domino Form:

New form: (interface content |FmContent), it be used to display data for the input interface.

New form: (ftp server configuration |FmFtpSet), it be used to configure the FTP servers of other application systems.

(3) Domino View :

Create view vwContent, the interface was used to display the existing list of data. 
Create vwFTP user FTP configuration documentation. New Page: \$ \$ viewtemplate for vwContent. It can be used to embellish the view vwContent.

(4) Resources:

New Image Resources: close.Jpg, editor.Jpg, save and exit.Jpg

(5)New Agent:

New Agent: agFtpDocOpen, to create one new system or modify other servers configuration information.

New Agent: DeleteDoc, to remove the documents, the parameters of this agent were the unid of transmission documents, to delete the corresponding documents.

New Agent: agDocSend, the agent was used to generate XML files and the corresponding attachments, and to upload those data to the servers of other application systems. This agent is the main function of this cross-platforms Interface. This function was achieved by using java agent. Three main classes were created: public class FtpUtil, public class JavaAgent extends AgentBase, public class MyXmlTool.

New agent: WebQueryOpen, it was used to invoked when opening the interface document, the main use is to display the operation toolbar and to provide different operating authority.

\section{Summary}

In this paper, One new cross-platform interface was researched and implemented to complete the data transmission between Domino-based application systems and other relational database-based application systems. The architecture of typical domino systems was discussed. For the practical needs, by using XML files as the carrier of structure data is one method to achieve data transmission between all application systems. And XML files were treated as an attachment, identified relations for the unstructured data transmission achievement. This interface, without connecting to the other database directly, uses XML as the carrier by using loosely coupled method to transmit structural and unstructured data. And it is a research and development direction of cross-platform data transmission.

\section{References}

[1] Guisset, Fabian. "What does each DOM Level bring?". Mozilla Developer Center. Mozilla Project. https://developer.mozilla.org/en/docs/DOM Levels. Retrieved January 10, 2011

[2] Akl, C.J.; Bayoumi, M.A.Single-Phase SP-Domino: A Limited-Switching Dynamic Circuit Technique for Low-Power Wide Fan-in Logic Gates, Circuits and Systems II: Express Briefs, IEEE Transactions on Volume: 55, 2008 , Page(s): $141-145$

[3] Fernandez-Valmayor, A.Building a Syntax Directed Processing Environment for XML Documents by Combining SAX and JavaCC, 2008. DEXA '08. 19th International Workshop on Database and Expert Systems Application 2008 , Page(s): 256 - 260

[4]Akl, C.J.; Bayoumi, M.A.Single-Phase SP-Domino: A Limited-Switching Dynamic Circuit Technique for Low-Power Wide Fan-in Logic Gates, Circuits and Systems II: Express Briefs, IEEE Transactions on Volume: 55, 2008 , Page(s): $141-145$

[5] Koch, Peter-Paul. "The Document Object Model: an Introduction". Digital Web Magazine. http://www.digital-web.com/articles/the document object model/. Retrieved January 10, 2009

[6] Thavot, R.; Bin Ab Rahman, A.A.-H.; Mosqueron, R.; Mattavelli, M.;Automatic mutli-connectivity interface generation for system designs based on a dataflow description, Ph.D. Research in Microelectronics and Electronics (PRIME),2010, Page(s): 1 - 4

[7] Joseph J, Bambara paul. J2EE Technology[M]. Beijing:China Machine Press. 2009:45 


\title{
Selection of Macroporous Resins for the Separation and Detection of
}

\section{Tomato Saponins}

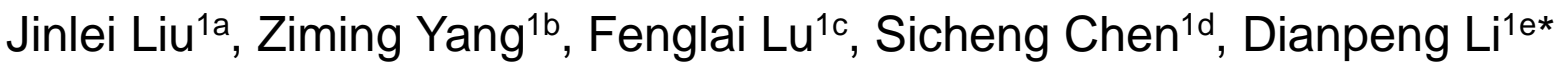 \\ agxibli|@163.com, ${ }^{b 786901683 @ q q . c o m},{ }^{\circ}$ 30575992@qq.com, ${ }^{d}$ csh@gxib.cn, eldp@gxib.cn
}

${ }^{1}$ Guangxi Key Laboratory of Functional Phytochemicals Research and Utilization, Guangxi Institute of Botany, Guilin 541006, China

Key words Tomato saponins; Macroporous resin; Adsorption and purification

Abstract This study was conducted to select a type of macroporous resin most suitable for the separation and purification of tomato saponins. The content of esculeoside A which is the major part of total tomato saponins was detected by colorimetry, and extraction rate of esculeoside A was used as an indicator to select the best type of macroporous resin for the isolation of tomato saponins. D101 has the highest adsorption efficiency for esculeoside A, with which, $0.25 \mathrm{~g}$ esculeoside A can be obtained from $1 \mathrm{~kg}$ tomato fruit.

Tomato (Lycopersicon esculentum Mill.), a member of the genus Lycopersicon in the family Solanaceae, is native to South America and widely planted in China nowadays. Two structurally complex steroidal saponins were isolated from fresh ripe tomato by Fujiwara from Kumamoto University, Japan in 2004, and named esculeoside $\mathrm{A}$ and esculeosideB ${ }^{[1]}$.Then, their physicochemical properties, physiological and pharmacological activities were systematically studied, and the results revealed that they were capable of reducing blood lipid, cholesterol, inhibiting cancer cell proliferation, anti-atherosclerosis, anti-oxidation and so on ${ }^{[2]}$. Therefore, esculeoside A and esculeoside B have attracted much attention of experts in cardiovascular diseases all over the world.

Macroporous resin is an organic adsorbent composed of high polymers and was developed in the 1960s. It has an internal three-dimensional stereoscopic pore structure, and good adsorption properties due to the numerous reticular cavities with a large specific surface area ${ }^{[3-4]}$. Macroporous resin can be used for purifying chemical substances from plant materials according to the polarity, relative molecular weight and different solubility of the objects ${ }^{[5]}$. In the present study, the adsorption capacity of 16 different types of macroporous resins to total tomato saponins were measured with spectrophotometry and compared to select the best macroporous resin type, and provide technical support for in-depth study of tomato saponins.

\section{Instruments and Materials}

\section{Materials}

Fresh ripe cherry tomatoes were purchased from the tomato planting base at Chetian Town, Ziyuan County, Guilin. Esculeoside A standard was purchased from Department of Pharmacy, Kumamoto University, Japan, and its mass fraction was 98.9\%, determined by HPLC. Sixteen types of macroporous resins: 1400, AB-8, CA-40, H103, HP-20, HPD100, HPD300, HPD400, HPD600, NKA, NKA2, NKA-9, S-8, Lukang 86021, Lukang DM130 and D101 were purchased from Tianjin Zhengtiancheng Resin Factory and Cangzhou Bao'en Adsorbing Material Technology Co., Ltd. Refined pectinase was purchased from Tianjin Lihua Enzyme Technology Co., Ltd. (with batch number: 20090603, food additive). 


\section{Instruments and regents}

T-6 spectrophotometer was purchased from Beijing Purkinje General Instrument Co., Ltd.; R-3 rotary evaporator was purchased from BUCHI Labortechnik AG; TL-5.0 desktop centrifuge was purchased from Shanghai Centrifuge Institute Co., Ltd.; TP-2200B electronic balance was purchased from Xiangyi Balance Instrument \& Equipment Co., Ltd.; METTLER AT200 analytical balance was purchased from Shanghai Hanjin Instrument \& Meter Co., Ltd.; vanillin, perchloric acid and glacial acetic acid were all of analytical grade; ethanol and methanol were chemically pure.

\section{Methods and Results}

\section{Pretreatment of macroporous resins}

Columns were filled with each type of macroporous resin $(200 \mathrm{~g})$, which had been soaked with $95 \%$ ethanol for $24 \mathrm{~h}$, eluted with $95 \%$ ethanol at a flow rate of $2 \mathrm{BV} / \mathrm{h}$, till the mixture of the elute and water was not cloudy any more. Then, the columns were washed with deionized water to remove ethanol, and before being washed with $5 \%$ dilute hydrochloric acid at a flow rate of $2 \mathrm{BV} / \mathrm{h}$ and soaked with it for $1 \mathrm{~h}$. Finally, the columns were washed with deionized water till the eluent's $\mathrm{pH}$ value was 7.0.

\section{Pretreatment of samples}

Thirty-four tomato samples were precisely weighed (1g each), washed and processed with a juicer. Then, the juice samples were digested with $0.5 \%$ pectinase at $50^{\circ} \mathrm{C}$ for $1.5 \mathrm{~h}$, filtered through gauze before centrifugation. The supernatant was stored in a refrigerator till analysis.

\section{Screening of the optimal macroporous resin}

Three copies of the 16 types of macroporous resin were precisely measured (150ml each copy) and loaded to columns. Then, the pretreated tomato samples were loaded to these columns to measure the adsorption capacity of the resins to total tomato saponins. After that, the columns were washed by 10 volumes of deionized water, and then eluted with $95 \%$ ethanol till the eluent was colorless. Finally, the eluents were concentrated under reduced pressure, vacuum-dried, and the resulting total tomato saponins were accurately weighed.

\section{Measurement of the content of total saponins in tomato}

\section{Preparation of standard solution of esculeoside $A$ and crude saponin solution}

5.0mg of esculeoside A standard was precisely weighed (with purity of 98.9\%), diluted with methanol to total volume of $5 \mathrm{ml}$, sonicated to obtain the standard solution of esculeoside A (1.0 $\mathrm{mg} / \mathrm{ml}$ ). $25.0 \mathrm{mg}$ of crude saponin powder was precisely weighed, diluted with methanol to total volume of $25 \mathrm{ml}$, sonicated, and filtered through microporous membrane to obtain the solution of crude saponins $(1.0 \mathrm{mg} / \mathrm{ml})$.

\section{Selection of measurement wavelength}

At first, $0.5 \mathrm{ml}$ of standard esculeoside A solution was transferred into a test tube with stopper, incubated in a water bath at $70^{\circ} \mathrm{C}$ till the solvent was evaporated to dryness. Then, $0.2 \mathrm{ml}$ of $5 \%$ vanillin - glacial acetic acid and $0.8 \mathrm{ml}$ of perchloric acid were added to the tube, mixed by shaking, and incubated in a water bath at $60^{\circ} \mathrm{C}$ for $20 \mathrm{~min}$. The tube was taken out of the water bath, allowed to stand at room temperature for $10 \mathrm{~min}$. $10 \mathrm{~min}$ after $5 \mathrm{ml}$ of glacial acetic acid was added, the tube was scanned at $400-700 \mathrm{~nm}$. The maximum absorption was found at 590nm. So, 590nm was considered as the wavelength most suitable for the determination of saponins.

\section{Establishment of standard curve}

$0.0,0.05,0.10,0.15,0.20,0.25$ and $0.30 \mathrm{ml}$ of standard esculeoside A solutions were precisely measured and transferred to $10 \mathrm{ml}$ tubes with stopper, incubated in a water bath at $70^{\circ} \mathrm{C}$ till the solvent was evaporated. Then, $0.2 \mathrm{ml}$ of newly prepared $5 \%$ vanillin - glacial acetic acid and $0.8 \mathrm{ml}$ 
of perchloric acid, were added to each tube, stoppered and mixed by shaking. The tubes were incubated in a water bath at $60^{\circ} \mathrm{C}$ for $20 \mathrm{~min}$, allowed to stand at room temperature for $10 \mathrm{~min}$. After $5 \mathrm{ml}$ of glacial acetic acid was added and mixed, the absorbance was read at 590nm. The standard curve was obtained by plotting absorbance on the Y-axis and the weight of esculeoside A on the $\mathrm{X}$-axis. The resulting regression equation was $Y=1.1088 X+0.0492, \mathrm{R}^{2}=0.996(n=6)$, wherein, $X$ is the weight of esculeoside $\mathrm{A}(\mathrm{mg})$ and $Y$ is absorbance. The equation revealed that there was a good linear relationship between absorbance and weight of esculeoside A (in a range of 0.052-0.312 $\mathrm{mg})$.

\section{Verification of experiment stability, accuracy and reproducibility}

$0.2 \mathrm{ml}$ of crude saponins solution prepared above was collected, processed as described in "Establishment of standard curve", and the absorbance was read 1, 2, 3, 4, 5, 6, 7 and 8h later. The RSD was $2.71 \%(n=8)$, indicating that the results were relatively stable within $8 \mathrm{~h}$. Six copies of standard esculeoside A solution $(0.2 \mathrm{ml})$, processed as described in "Establishment of standard curve", and the absorbance was read 1, 2, 3, 4, 5, 6, 7 and $8 \mathrm{~h}$ later. The $R S D$ value was $1.11 \%(n=6)$, suggesting that this method was accurate. Six copies of crude saponin powder (10mg each), diluted with methanol to a total volume of $10 \mathrm{ml} .0 .2 \mathrm{ml}$ of the solution, and its absorbance was measured following the steps described above. The resulting $R S D$ value was $1.14 \%(n=6)$, indicating that the method was repeatable.

Table1 The stability, accuracy and reproducibility of the system we developed

\begin{tabular}{clll}
\hline & Stability & Accuracy & Reproducibility \\
\hline 0.146 & 0.297 & 0.155 \\
0.148 & 0.290 & 0.156 \\
& 0.153 & 0.291 & 0.153 \\
Absorbance (A) & 0.155 & 0.299 & 0.157 \\
& 0.16 & 0.293 & 0.156 \\
& 0.149 & 0.292 & 0.152 \\
\hline$R S D(\%)$ & 0.152 & & \\
\hline
\end{tabular}

\section{Test on recovery rate of saponins}

Four copies of crude saponins powder samples, in which the esculeoside A content was measured, (about 14\%) were precisely weighed (100 mg each copy). Four copies of esculeoside A standard were also precisely weighed ( $2 \mathrm{mg}$ each) and added to the four copies of crude saponins powder samples. Then, each sample was diluted to $50 \mathrm{ml}$. The peak area was measured to calculate the recovery rate, average recovery rate and $R S D$ value. All the data revealed that the recovery rate and $R S D$ value met the requirements as expected.

Measured weight of esculeoside $\mathrm{A} / \mathrm{mg} \div$ (the weight of esculeoside $\mathrm{A}$ in sample/mg + the weight of esculeoside A added to sample $/ \mathrm{mg}) \times 100 \%=$ recovery rate 
Table 2 Test on recovery rate

\begin{tabular}{lllllll}
\hline $\begin{array}{l}\text { Crude saponins } \\
(\mathrm{mg})\end{array}$ & $\begin{array}{l}\text { Esculeoside A in } \\
\text { crude sample }(\mathrm{mg})\end{array}$ & $\begin{array}{l}\text { Esculeoside } \\
\text { added to sample } \\
(\mathrm{mg})\end{array}$ & $\begin{array}{l}\text { Measured weight of } \\
\text { esculeoside A }(\mathrm{mg})\end{array}$ & $\begin{array}{l}\text { Recovery } \\
\text { rate }(\%)\end{array}$ & $\begin{array}{l}\text { Mean of } \\
\text { recovery } \\
\text { rate }(\%)\end{array}$ & $\begin{array}{l}\text { RSD } \\
(\%)\end{array}$ \\
\hline 101.92 & 11.19 & 1.98 & 12.74 & 95.86 & 96.65 & 96.03 \\
101.30 & 11.12 & 1.95 & 12.66 & 95.58 & 0.41 \\
101.20 & 11.11 & 2.28 & 12.48 & 96.02 & \\
100.70 & 11.05 & & & &
\end{tabular}

Table 3 Adsorption capacity of different types of macroporous resins to esculeoside A

\begin{tabular}{|c|c|c|c|c|}
\hline No. & Macroporous resin & Weight of crude saponins (g) & Weight of esculeoside A (g) & $\begin{array}{l}\text { Percentage of esculeoside } \\
\text { A }(\%)\end{array}$ \\
\hline 1 & D-101 & 1.90 & 0.2381 & 12.53 \\
\hline 2 & AB-8 & 2.02 & 0.2117 & 10.48 \\
\hline 3 & HP-20 & 2.17 & 0.2114 & 9.74 \\
\hline 4 & HPD-600 & 1.84 & 0.2077 & 11.29 \\
\hline 5 & HPD-400 & 1.79 & 0.2042 & 11.41 \\
\hline 6 & Lukang-86021 & 1.66 & 0.2037 & 12.27 \\
\hline 7 & HPD-300 & 2.04 & 0.2034 & 9.97 \\
\hline 8 & HPD-100 & 1.97 & 0.2013 & 10.22 \\
\hline 9 & $\mathrm{CA}-40$ & 1.73 & 0.1953 & 11.29 \\
\hline 10 & $\mathrm{H}-103$ & 2.52 & 0.1887 & 7.49 \\
\hline 11 & NKA & 1.94 & 0.1862 & 9.60 \\
\hline 12 & $\mathrm{~S}-8$ & 1.12 & 0.1858 & 16.59 \\
\hline 13 & Lukang-DM130 & 1.81 & 0.1855 & 10.25 \\
\hline 14 & NKA-9 & 0.99 & 0.1817 & 18.35 \\
\hline 15 & 1400 & 1.89 & 0.1724 & 9.12 \\
\hline 16 & NKA-2 & 0.90 & 0.0140 & 1.56 \\
\hline
\end{tabular}

\section{Determination of the content of total saponins in samples}

The standard esculeoside A solution and tomato sample solution prepared above were pipetted $\left(0.5 \mathrm{ml}\right.$ each) and transferred to test tubes with stopper, incubated in a water bath at $70^{\circ} \mathrm{C}$ till the solvent was evaporated to dryness. Then, $0.2 \mathrm{ml}$ of $5 \%$ vanillin - glacial acetic acid and $0.8 \mathrm{ml}$ of perchloric acid were added to each tube, mixed by shaking, incubated in a water bath at $60^{\circ} \mathrm{C}$ for $20 \mathrm{~min}$. The reaction was stopped by incubating in ice for $10 \mathrm{~min}$. Ten minutes after $5 \mathrm{ml}$ of glacial acetic acid was added to each tube, the absorbance was read at $590 \mathrm{~nm}$. Finally, the content of total tomato saponins in samples was calculated from absorbance according to the standard curve obtained above. 


\section{Results and Analysis}

As shown in Table 3, among all types of macroporous resin, D-101 had the best absorption capacity to esculeoside A, followed by AB-8. NKA-9 was most specific to esculeoside $A$ and thus the resulting percentage of esculeoside A was the largest, although it had a lower absorption capacity. In addition, D-101 and AB-8 were two types of macroporous resin most commonly used in previous studies for the extraction of saponins, so there is a need to determine the optimal parameters for saponin extraction.

\section{Discussion}

Currently, saponins in ginseng, bitter gourd, yam and other plants have been studied a lot, but saponins in tomato has been rarely reported. It has been proven that saponins have a variety of physiological and pharmacological activity ${ }^{[6-7]}$. We have found that tomato saponins has a unique role in lipid-lowering and anti-atherosclerosis. It can regulate lipid metabolism, lower blood viscosity, increase the capacity of oxidative free radical scavenging, reduce lipid peroxide, which prevents and suppresses the formation of atherosclerosis ${ }^{[8]}$. Moreover, the water extract of tomato saponins can effectively regulate lipid metabolism in human bodies, and thus can be used for the treatment of hyperlipidemia ${ }^{[9]}$. Macroporous resins have been widely used for the isolation and purification of saponins, alkaloids, flavonoids, peptides, carbohydrates and other chemicals ${ }^{[10]}$. In this study, the macroporous resin type most suitable for the isolation of tomato saponins was determined, which will provide a technical support for in-depth study of saponins.

Tomato saponins have no absorption in UV region. It will need a capital investment in instruments using evaporative light scattering derector to detect them. Vanillin colorimetry is simple, low-cost, and reliable, but the results are greatly affected by temperature and reaction time. So the reaction conditions should be strictly controlled and three or more repetitions should be prepared when vanillin colorimetry is adopted for the measurement of tomato saponins.

\section{Acknowledgements}

This work was supported by Agricultural science and Technology Achievements Transformation Fund Project(2014GB2E100274); Fund of Guangxi Key Laboratory of Functional Phytochemicals Research and Utilization (ZRJJ2012-6); Supported by Bagui Scholar Program of Guangxi (Guangxi Institute of Botany ).

\section{References}

[1] Yukio Fujiwara, Naoko Kiyota, Masaharu Hori, Sayaka Matsushita, Yoko Iijima, Koh Aoki, Daisuke Shibata, Motohiro Takeya, Tsuyoshi Ikeda, Toshihiro Nohara, Ryoji Nagai. American Heart Association, 2007, (27): 2400-2406.

[2] Friedman M , Levin CE , Lee SU , Kim HJ , Lee IS , Byun JO , Kozukue N. Agricultural and Food Chemistry, 2009, (57): 5727-5733.

[3] Maotian Wang, Shan Pei. Chemical Industry Press , 2004: 100-105. (In Chinese)

[4]Chenghe Sun, Yingping Wang, Shiying Zhao Special Wild Economic Animal and Plant Research , 2008, (2): 55-57. (In Chinese)

[5] Xiaomin Li , Xuan Wu, Zhongliang Xiong . Journal of Chinese Medicinal Materials, 2009, 10(32): 1613 -1615.(In Chinese)

[6] Ting Jin . Food Engineering, 2008, (1): 19-21.(In Chinese) 
[7] Yongxia Tao, Jihua Duan . Academic Periodical of Farm Products Processing, 2007, (6): 84-85. (In Chinese)

[8] Jianzhang Wu, CiyuLi, Jinlei Liu . Food Research and Development, 2011, (11): 131-134. (In Chinese)

[9] Jianzhang Wu, CiyuLi, Jinlei Liu . Food Research and Development, 2011, (3): 167-170.(In Chinese)

[10] Haixia Zhao , Jian Tong, China Food Additives, 2009, (7): 131-134. (In Chinese) 


\title{
Development of a Precise Broken End Positioning and Indicating System
}

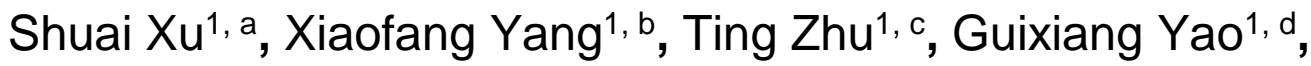 \\ and Chunmo Wang ${ }^{1, e}$
}

${ }^{1}$ No. 285 South Jiefang Rd. Yancheng Institute of Industry Technology, Yancheng(224005), Jiangsu, China

axushuai430@126.com, byxf1984ok@126.com, cleon_china@163.com, dyaoguixiang8067199@126.com, ${ }^{\mathrm{e}}$ wangchunmo@126.com

\begin{abstract}
Keywords: warp break detector, precise positioning, dropper bar, resistance measurement
Abstract: Modern looms can usually detect a broken warp yarn by using a warp break detector, but they cannot locate the broken end precisely and automatically. High-count and high-density fabrics with a broad width usually have a warp consisting of up to ten thousand of ends that are closely packed. This makes it very difficult for a worker to locate the broken end quickly. In order to solve this problem, the authors developed a precise broken end positioning and indicating system. It has advantages such as high operational stability, high positioning accuracy, and clear visual indication of failures.
\end{abstract}

\section{Forewords}

Most modern looms have a warp stop motion to stop the machine immediately when a warp yarn is broken. Currently, a warp break detector of the warp stop motion can detect any broken warp yarn by using the droppers, but it cannot visually show its location and a worker has to locate it manually. For the high-count and high-density fabric with a broad width, as there can be up to ten thousand warp ends, it is even more difficult to find the position of the broken end. In order to solve this problem, the authors developed a new system to precisely locate the broken ends.

\section{The Principle of Positioning}

\section{Basic Principles of Warp Break Detector}

The most commonly used contact warp break detector consists of a dropper bar and droppers. The structure of a typical dropper bar is shown in Fig. 1. It is composed of an electrode 1, an electrode 2 and an insulating layer 3 . The insulating layer 3 is between the electrode 1 and the electrode 2 and it separates them, so the electrode 1 and electrode 2 are insulative to each other.

Fig. 2 shows the operating principle of the contact warp break detector. When a warp end 5 is broken or becomes too loose, the dropper falls and electrode 1 and electrode 2 become conductive to each other. At this time, a signal is sent out and the loom stops immediately. Electrode 1 is mostly made of brass, and the electrode 2 and dropper 4 are made of stainless steel.

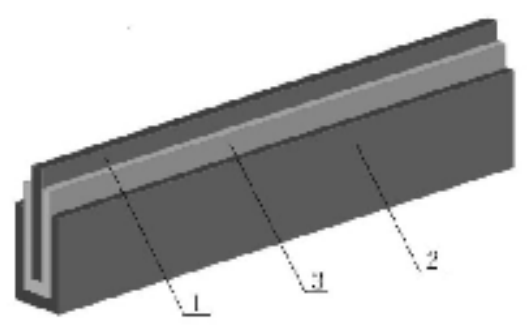

Fig. 1. Structure of dropper bar

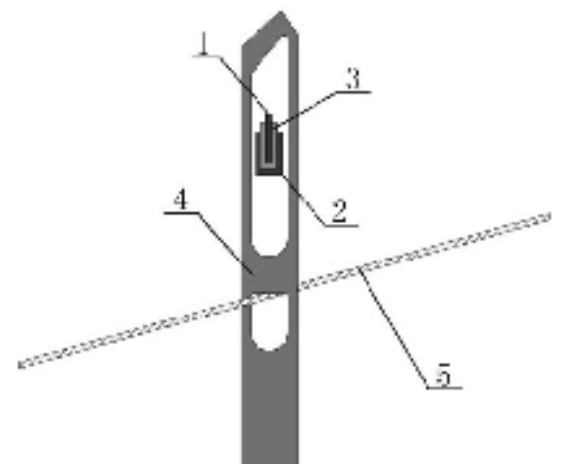

Fig. 2. Operating principle of contact warp break detector Principle of Resistance Positioning 
The model of a contact warp break detector can be simplified as shown in Fig. 3(a). When the electrode 1 and electrode 2 become conductive by the dropper 4, the distance between dropper 4 and the loom's right end and the left end are marked as $\mathrm{X}$ and $\mathrm{Y}$ respectively. Then, the corresponding electric circuit diagram of Fig. 3(a) is shown in Fig. 3(b). In Fig. 3(b), $R_{\mathrm{C} 1}$ and $\mathrm{R}_{\mathrm{C} 2}$ represent the contact resistances between the dropper 4 and the electrode 1, and the dropper 4 and the electrode 2 respectively. $R_{W}$ is the resistance of the dropper 4 itself. $R_{1 X}$ is the resistance produced by the part $X$ of the electrode 1 , and $R_{1 Y}$ is produced by part $Y$. Similarly, $R_{2 X}$ and $R_{2 Y}$ are created in the same way. If any of $R_{1 X}, R_{1 Y}, R_{2 X}$ and $R_{2 Y}$ 's values is obtained, the distances $X$ and $Y$ can be calculated by the law of resistance, e.g. the value of $R_{2 X}$ is obtained, the distance $X$ can be calculated and determined as follows.

Let electrode 2's resistivity be $\rho$, cross section area be $S$, then there is:

$$
R_{2 X}=\frac{\rho X}{S}=k X
$$

In the formula, $k$ can be treated as unit length resistance of a material and it can be measured. Its unit is $\Omega / \mathrm{m}$. So, the value of $\mathrm{X}$ would be:

$$
L=\frac{R_{2 X}}{k}
$$

If the formula is further analyzed, we come to a conclusion that in order to make the $\mathrm{X}$ accurate, $k$ and $\mathrm{R}_{2 \mathrm{X}}$ have to be accurate in the first place.

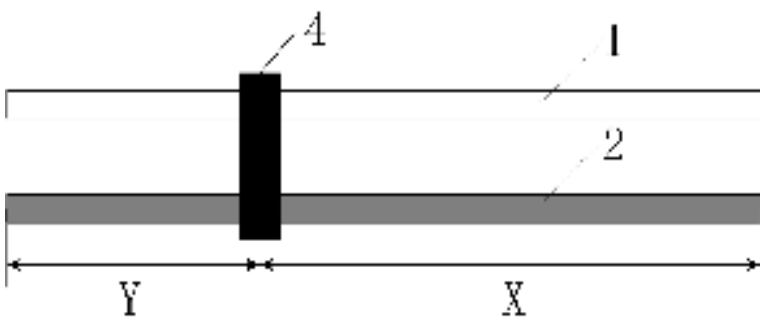

(a) model

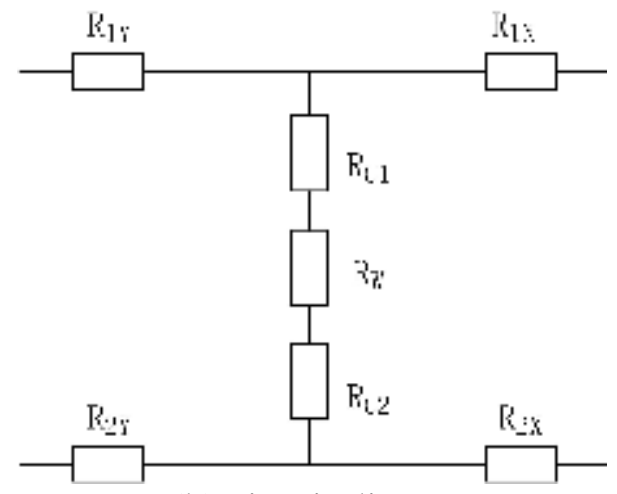

(b) circuit diagram

Fig. 3. Model and circuit diagram of warp break detector

\section{Design of a New End Break Detector}

To design a precise broken end positioning and indicating system, the structure of the warp break detector must be redesigned to ensure the values of $\mathrm{k}$ and $\mathrm{R}_{2 \mathrm{X}}$ are as reliable as possible. And after that, a matching resistance measuring system can be designed. The value of $\mathrm{k}$ needs to be constant and cannot be too small. The reason of that is explained as follows.

Suppose in one measurement, the $\mathrm{R}_{2 \mathrm{x}}$ in Fig. 3 is measured $R i$, and the error margin is $\Delta R$. The actual value is $R_{0}$, which equals the resistance value of length $X_{0}$ of electrode 2 . Then we come to the following formula (3).

$$
R_{i}=R_{0}+\Delta R
$$

Based on formulas (2) and (3), let the measured length be $X i$, the value of $X i$ will be:

$$
\mathrm{X}_{i}=\frac{R_{i}}{k}=\frac{R_{0}+\Delta R}{k}=X_{0}+\frac{\Delta R}{k}
$$

If $X i$ is used as the final result to determine the position of the broken end, the error will be:

$$
\Delta X=X_{i}-X_{0}=\frac{\Delta R}{k}
$$


It is generally known that the error of a measurement is random and has a constant range, and it will not dwindle down as the measured values get smaller. From formula (5), the smaller $k$ is, the greater the positioning error will be. Besides, it is more difficult to get an accurate measurement for smaller resistances. For these reasons the choice of electrode depends upon the material's $k$ value, i.e. the unit length resistance. And a greater $k$ value will be better to the system.

As mentioned above, currently, the materials of the dropper bar are brass and stainless steel, and the $k$ is very small. This leads to the fact that the resistance is only $1-2 \Omega$ for the full length of the electrode, which is usually 2-4 meters long. This will be a disadvantage for measuring the resistance. Therefore, changes must be made to the materials and structure of the electrode. Fig. 4 shows the structure of the newly designed dropper bar. The material for electrode 1 remains unchanged, brass, However, the electrode 2, which is attached to the insulating layer 3, no longer uses stainless steel. Instead, a $0.1 \mathrm{~mm}$ diameter $\mathrm{Cr} 20 \mathrm{Ni} 80$ nichrome wire is used. The wire's resistance per unit length is $138.8 \Omega / \mathrm{m}$. It also has good physical properties such as good resistance to abrasion and corrosion, which make it ideal for the new positioning system.

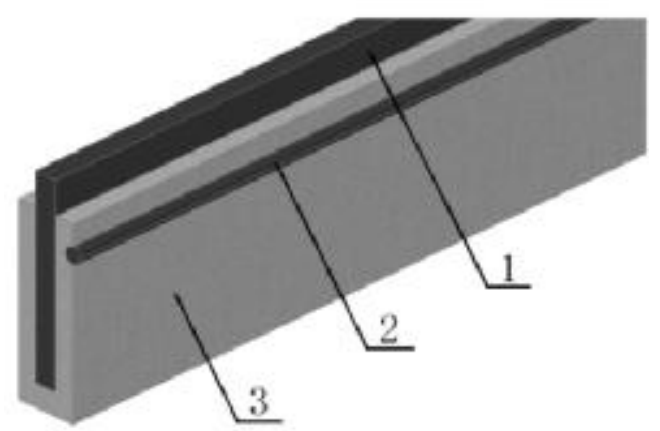

Fig. 4. Structure of new dropper bar

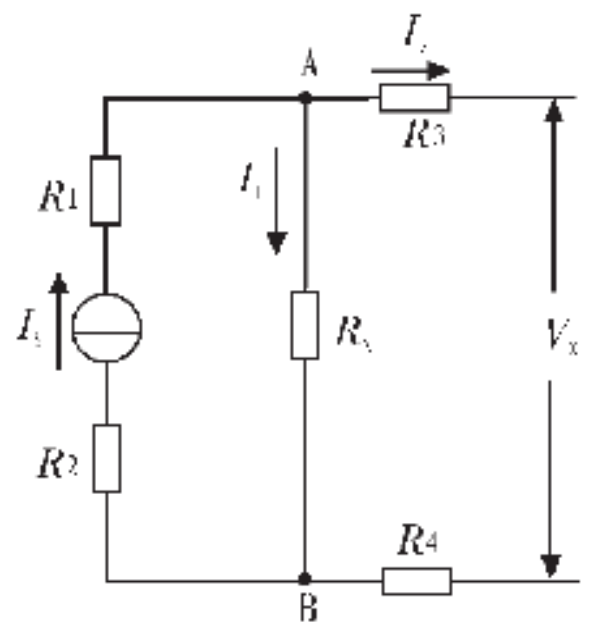

Fig. 5. Four-wire method

\section{Design of the System}

\section{Methods of Resistance Measurement}

The model and the circuit diagram shown in Fig. 3 can still be used for the new dropper bar shown in Fig. 4. Now obtaining the accurate resistance value for $R_{2 x}$ is crucial to precise positioning. Because the dropper bar is full of droppers when in operation, it would be difficult to directly connect two wires to both ends of $R_{2 x}$. If it is directly measured from the right end, errors will occur because resistance values of $R_{C 1}, R_{C 2}, R_{W}$ and $R_{1 X}$ are also included. In order to solve this problem, this paper adopts the four-wire method, which is generally accepted as the best way to measure resistance. The principle of the four-wire method is shown in Fig. 5.

In Fig. 5, Is is a constant current source. Rx is the resistance to be measured. A and B are the two voltage measuring points. $R_{1}, R_{2}, R_{3}$ and $R_{4}$ are the resistances of the four wires. From Fig. 5 , we have formula (6).

$$
I_{S}=I_{1}+I_{2}
$$

When the signal is amplified by an instrumentation amplifier, the input impedance of the amplifier is extremely high and its bias current is extremely small, that means in formula (6): $I_{2} \approx 0, I_{S} \approx I_{1}$.

Because $\mathrm{I}_{2} \approx 0$, the decrease of voltage caused by the wires $\mathrm{R}_{3}$ and $\mathrm{R}_{4}$ is negligible. So $\mathrm{V}_{\mathrm{X}}$ equals the voltage on $R_{X}$. From this, the accurate value of $R_{X}$ can be calculated by $R_{X}=V_{X} / I s$.

Similarly, the new method for resistance measurement based on the four-wire method is shown in Fig. 6. Although the resistances of $R_{C 1}, R_{C 2}, R_{W}$ and $R_{1 x}$ are unknown, the incurring voltage decrease 
of them can be neglected for the same reason discussed above. Finally, we come to the conclusion that the input voltage on the instrumentation amplifier is exactly equal to the voltage of $R_{2 X}$.

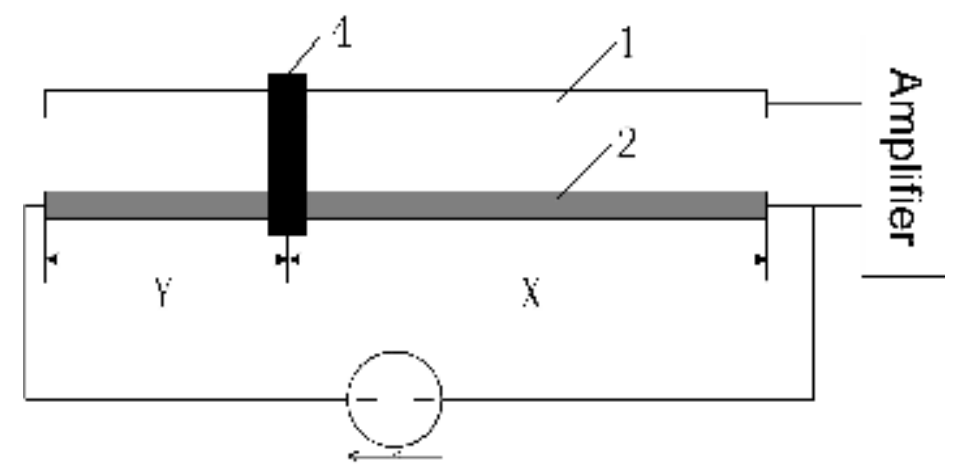

Fig. 6. Voltage measurement of $\mathrm{R}_{2 \mathrm{X}}$

\section{Overall Design of the System}

The overall structure of the system is shown in Fig. 7. Below is how it works. First, the system uses the original circuit and photocoupler of the loom, which are used to check if there is a broken end. When a broken end is detected, the power of the checking circuit is cut by the relay. Meanwhile, the relay provides power to the constant current source and the signal processing circuit, which makes it possible to measure the resistance. After that, the resistance is converted to a distance between the broken end and the right end of the loom by the MCU, and finally the position of the broken end is determined based on the distance and is shown by an indicator.

The function of the relay is to switch between two directions of power supply, one is to the original checking circuit and the other is to the constant current source circuit and the signal processing circuit. The circuits of the two directions are separated and do not interfere with each other. Besides, as shown in Fig. 7, the checking circuit's wire is connected to the left end of the bar, and the signal processing circuit's wire is connected to the right end.

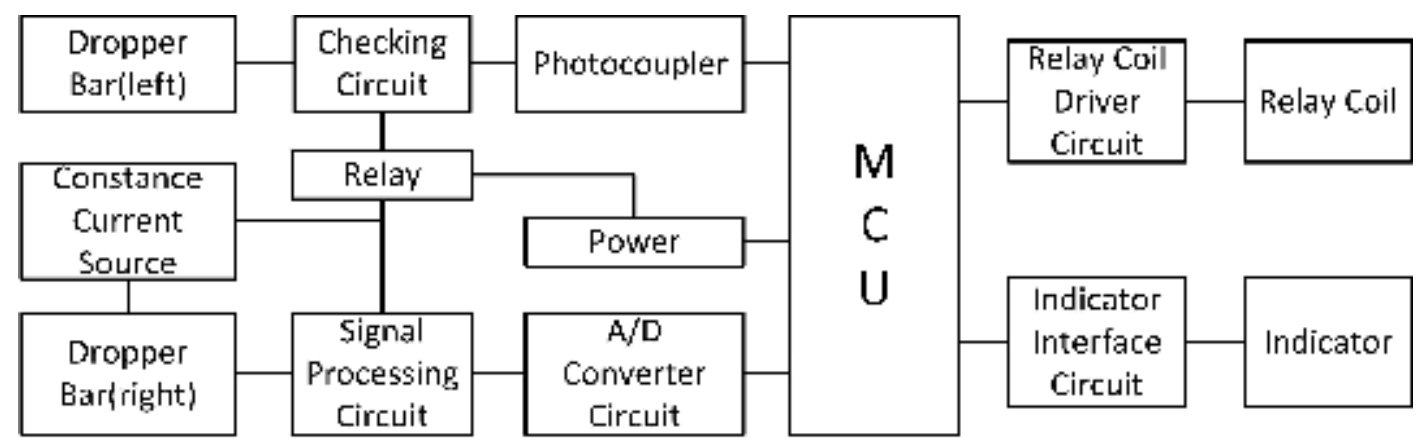

Fig. 7. System structure

\section{Design of System Hardware}

\section{Design of Constant Current Source Circuit}

The constant current source of the system mainly consists of an ultralow offset voltage operational amplifier OP07 and a high precision resistor. The amplifier's control voltage is from the REF pin of ADC MAX197. The constant current circuit and A/D converter circuit use the same reference voltage, which means the drift of reference voltage does not affect the measurements, and the designing of the constant current source can be simplified. In the constant current circuit, shown in Fig. 8, the current is calculated as $\mathrm{I}=4.096 \mathrm{~V} / 2 \mathrm{k} \Omega=2.048 \mathrm{~mA}$. As the resistor's temperature coefficient and precision will significantly affect the circuit, a high precision resistor is recommended here. 


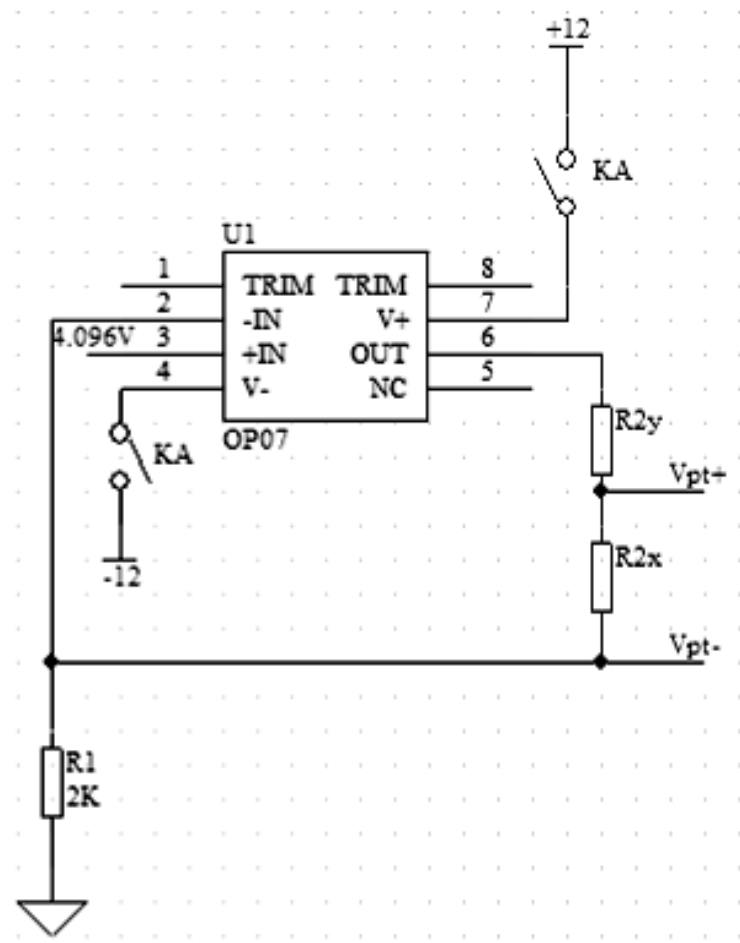

Fig. 8. Constant current source circuit

\section{Design of Signal Processing Circuit}

As shown in Fig. 9, the signal processing circuit in this design involves two operational amplifiers, AD620 and OP07. AD620 is an instrumentation amplifier with high precision and stability. Its input impedance is up to $10 \mathrm{G} \Omega$, and the bias current is as low as $2 \mathrm{nA}$. The gain setting of AD620 is as simple as adding an external resistor $R_{G}$ between pin 1 and pin 8 . The gain equation is $G=1+$ $49.4 \mathrm{k} \Omega / \mathrm{R}_{\mathrm{G}}$. The length of the dropper bar varies on different types of looms, but is usually less than four meters, which makes the value of $\mathrm{R}_{2 \mathrm{X}}$ in Fig. 3 fall in $0 \Omega \sim 555.2 \Omega$. And in the constant current source circuit, the voltage of $\mathrm{R}_{2 \mathrm{x}}$, i.e. the input voltage of signal processing circuit, is $0 \mathrm{mV} \sim 1137 \mathrm{mV}$. However, the output voltage of signal processing circuit must be $0 \mathrm{~V} \sim 5 \mathrm{~V}$. For this reason, the voltage needs to be converted by a proper gain $\mathrm{G}$. That is, $\mathrm{G}=5 / 1.137 \approx 4.4$.

\section{Design of A/D Converter Circuit}

The A/D converter used in the design is a 8-channel, 12-bit converter MAX 197. The model of the MCU is AT89S52. The connection of them is shown in Fig. 10. MAX 197 runs in internal clock mode in this design and uses the internal reference. REF pin outputs a reference voltage of 4.096v that is used as the control voltage by the constant current source.

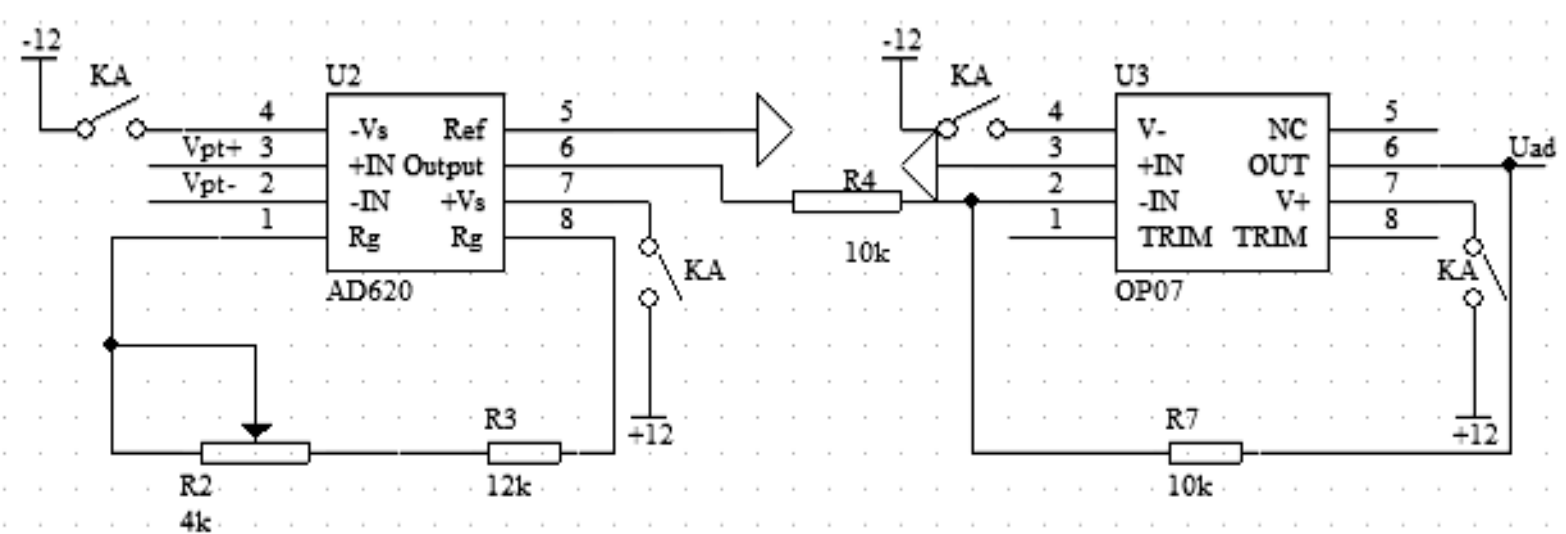

Fig. 9. Signal processing circuit 


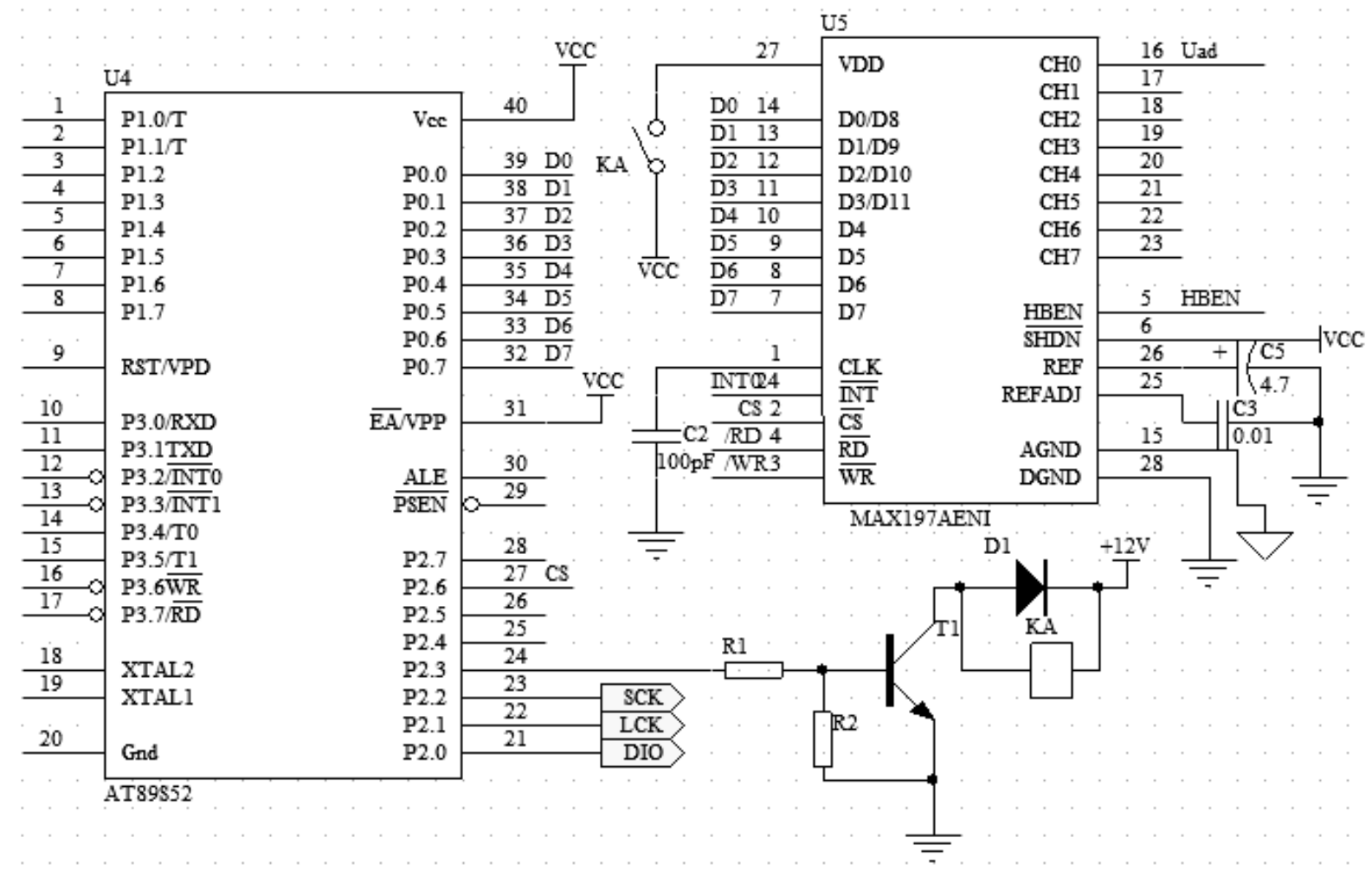

Fig. 10. A/D converter circuit

\section{Design of Indicator Circuit}

The indicator of the system is a long PCB with a row of LED bulbs on it, and its length equals the length of the dropper bar. All the LEDs on the PCB are spaced $1 \mathrm{~cm}$ apart to indicate the position of the broken end. Considering the length of the dropper bar on most looms, the number of LEDs of the indicator ranges from 200 to 400. In order to control all these LEDs, two 74HC595 registers are used to create an extendable indicator, of which the length is adjustable. The connection of $74 \mathrm{HC} 595$ registers and the LEDs is shown in Fig. 11. In the figure, two 74HC595 registers control 64 LEDs. Both the registers and the LEDs are installed on a $64 \mathrm{~cm}$ long PCB, which can be extended according to the length of the dropper bar.

\section{Design of System Software}

The difficulty of system software designing is how to timely switch between the checking program which is used to determine if there is a broken end and the positioning program which is used to locate it. The flow chart of the program is shown in Fig. 12. When the program runs, a flag bit is created to control the relay. It is also used to prevent the relay from operating again after a broken end has been located and displayed. The flag bit and the indicator are initialized by an interrupt program when the start button of the loom is pressed. Besides, the A/D conversion process in the program is supposed to be executed multiple times in order to get better results.

In the distance calculation, as mentioned earlier, the maximum length of the dropper bar is four meters, and the PCB has the same length with 400 LEDs on it. Suppose the result of A/D conversion is $V_{0}$ in a measurement, then the $X$ in the Fig. 3 is calculated by the formula $X=V_{0} \times 400 / 4096$, and the unit of $\mathrm{X}$ is $\mathrm{cm}$. As $\mathrm{X}$ represents the distance between the broken end and the right end of the dropper bar, the LED for the broken end is determined and lights up. 

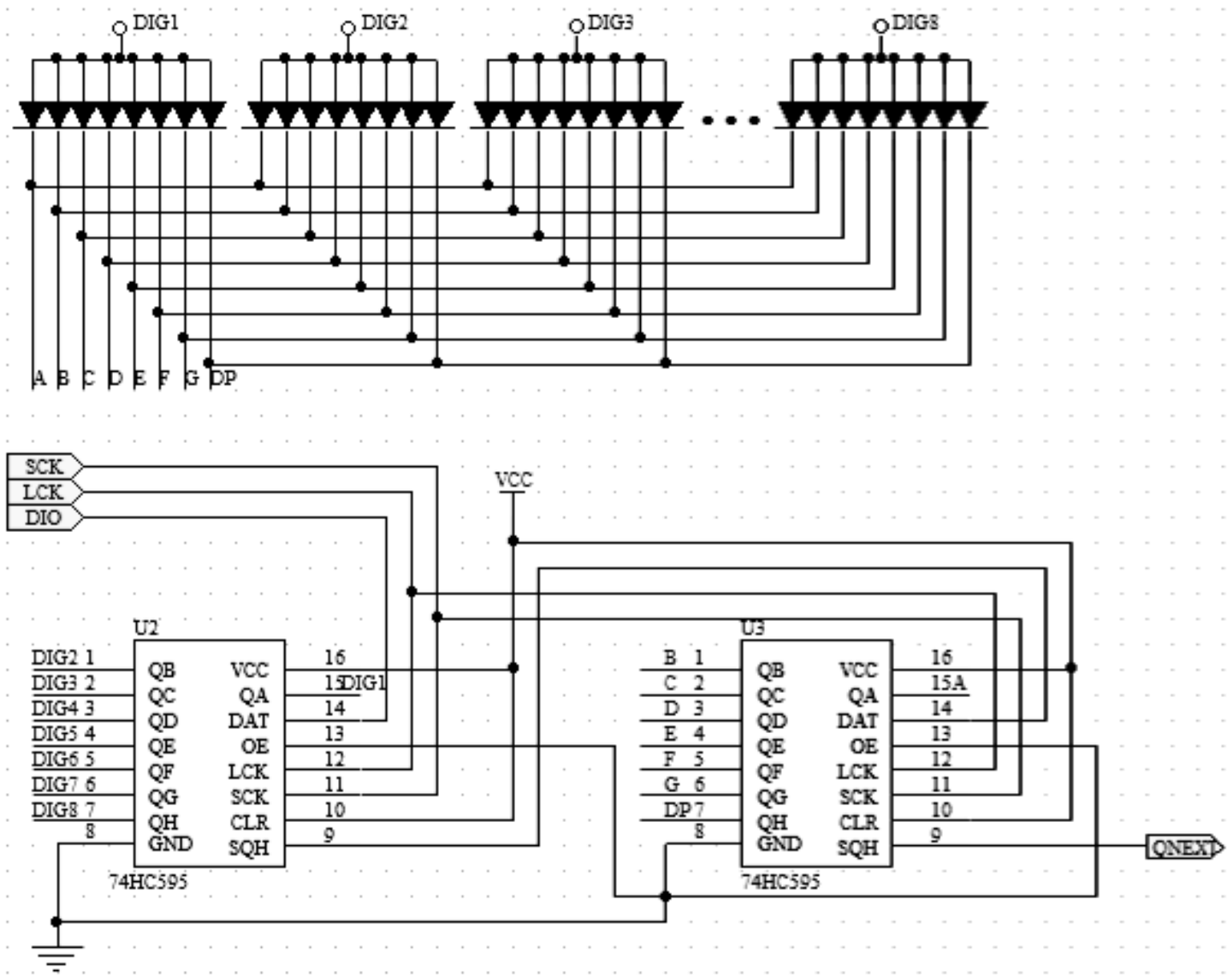

Fig. 11. Indicator circuit

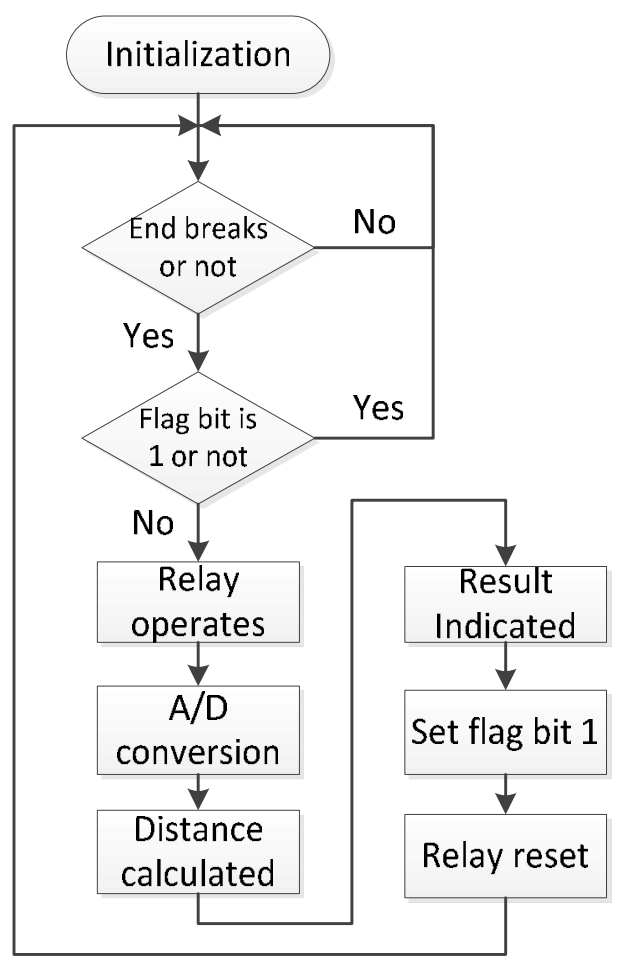

Fig. 12. Flow chart of the program 


\section{Conclusions}

The system works on the basis of law of resistance, uses a nichrome wire as the electrode and adopts the four-wire method for resistance measurement. The system includes a constant current source based on the ADC reference, a high-precision instrumentation amplifier, and an extendable LED indicator. The system runs stably and indicates visually. Practical applications have shown that the system is able to precisely locate the broken end, and therefore it significantly improves the operation efficiency of the workers and reduces damage to other warp ends.

\section{References}

[1] Zhang Xiutai, Hu Xuehui, Zhai Yafang: Chinese Journal of Sensors and Actuators. Vol. 23 No.6 (June 2010), p. 812-815.

[2] LIU Yun, LIN Xue-yan: Electromechanical Components, 6(2) (2008), p. 23-26.

[3] SUNAMID, MCNAMESJ, COLBERTA, etal. Variable frequency bioimpedance instrumentation[C]. Proceeding 26th Ann. Inter. Conf. IEEE Eng. Med. Biol. Soc., 2004(4):2386-2389..

[4] ROSSA S. Current source design for electrical impedance tomography[J]. Physiological Measurement, 2003, 24(2):509-516.

[5] LEEJW. Precision constant current source for electrical impedance tomography[J]. Proceeding of 25th Annual International Conference of the IEEE EMBS, Cancun, Mexico, 2003:17-21.

[6] Zhao Shaobo: Chinese Journal of Scientific Instrument, Vol. 22 No.4 (August 2001), p. $136-138$

\section{Acknowledgements}

This work was financially supported by Industry-university-research Cooperation Prospective Project of Jiangsu (BY2014112) and Top-notch Academic Programs Project of Jiangsu Higher Education (PPZY2015C254). 


\title{
Analysis of Ceramic Pattern Primitives Transform Operation
}

\author{
LI Busheng ${ }^{1}, \mathrm{HU}$ Jingfang ${ }^{2}$ \\ 1,2School of Information Engineering, Jingdezhen Ceramic Institute, Jiangxi, P.R.China \\ 1abulbs@163.com, 2idzhjf@163.com
}

Keywords: ceramic pattern; geometric transformation; Entity

Abstract. This paper analyzes the entity atomic operations include translation, scaling, rotation, mirror, waveform transform and vortex transformation mainly six geometric transformation, and according to the characteristics of ceramic pattern, has carried on the experiment to verify the analysis.

\section{Introduction}

Geometric arithmetic of image can change the spatial relationship between objects in an image. A geometric arithmetic requires two independent algorithms. The first, it need an algorithm to define space transform itself, describe how each pixel from the initial position to 'move' to terminate the position with it, namely each pixel 'move-ment'. Second, it also requires a gray interpolation algorithm. Because, the position coordinates of the input image $(\mathrm{x}, \mathrm{y})$ is an integer, but the position coordinates of the output image is not integer, the converse is also true.

In most applications, connectivity requirements keep curve features in the image of the continuity and the object. An unconstrained spatial transform algorithm is likely to break line and broken images. In general we use mathematical methods to describe the spatial relationship between the input and output image point. The general definition for geometric arithmetic:

$$
g(x, y)=f\left(x^{\prime}, y^{\prime}\right)=f[a(x, y), b(x, y)]
$$

Wherein, $f(x, y)$ represents the input image, $g(x, y)$ represents the output image. The function $a(x$, $y)$ and $b(x, y)$ only describes the spatial transformation. If they are continuous, then the continuous relationship will be maintained in the image [1].

\section{The Geometric Transformation of Graphic}

The first requirement of geometric transformation is to run an algorithm which moves the pixel from a position to another position. We introduce several common geometric transformations.

Through the previous introduction, we are ready for the geometric transformation of the image. In equation (1), if

$$
a(x, y)=x^{\prime} \text { And } b(x, y)=y^{\prime}
$$

Then we get an identity arithmetic simply copy f to $\mathrm{G}$ without any changes.

\section{A Translation}

If makes the $a(x, y)=x+x O$, and $b(x, y)=y+y 0$ then it get a translation arithmetic, the point $(x 0, y 0)$ is translated to the origin, and the feature points in the image move $\sqrt{\left(x_{0}{ }^{2}+y_{0}^{2}\right)}$. Using the 
homogeneous coordinate expression way, we can think the plane $x y$ is a plane which $\mathrm{Z}=0$ in the three-dimensional space $x, y, z$. And the equation (2) expressed in concise matrix as follows:

$$
\left(\begin{array}{l}
a(x, y) \\
b(x, y) \\
1
\end{array}\right)=\left(\begin{array}{ccc}
1 & 0 & x 0 \\
0 & 1 & y 0 \\
0 & 0 & 1
\end{array}\right)\left(\begin{array}{l}
x \\
y \\
1
\end{array}\right)
$$

\section{B Scaling}

If the $a(x, y)=x / c, B(x, y)=y / d$, it will make the image magnified $c$ times in the $X$ direction, magnified $\mathrm{D}$ times in the direction of $\mathrm{Y}$ axis. The origins of image keep still when the image is magnified.

$$
\left(\begin{array}{l}
a(x, y) \\
b(x, y) \\
1
\end{array}\right)=\left(\begin{array}{ccc}
1 / c & 0 & x 0 \\
0 & 1 / d & y 0 \\
0 & 0 & 1
\end{array}\right)\left(\begin{array}{l}
x \\
y \\
1
\end{array}\right)
$$

\section{Mirror}

If the $\mathrm{c}=1$, it will produce a $\mathrm{Y}$ axis symmetric image $\mathrm{a}(\mathrm{x}, \mathrm{y})=-\mathrm{x}, \mathrm{b}(\mathrm{x}, \mathrm{y})=\mathrm{y}$.

$$
\left(\begin{array}{l}
a(x, y) \\
b(x, y) \\
1
\end{array}\right)=\left(\begin{array}{ccc}
-1 & 0 & 0 \\
0 & 1 & 0 \\
0 & 0 & 1
\end{array}\right)\left(\begin{array}{l}
x \\
y \\
1
\end{array}\right)
$$

Similarly, also have the same properties for $\mathrm{D}$ and $\mathrm{X}$.

$$
\left(\begin{array}{l}
a(x, y) \\
b(x, y) \\
1
\end{array}\right)=\left(\begin{array}{ccc}
1 & 0 & 0 \\
0 & -1 & 0 \\
0 & 0 & 1
\end{array}\right)\left(\begin{array}{l}
x \\
y \\
1
\end{array}\right)
$$

\section{Rotation}

Let $a(x, y)=x \cos (\theta)-y \sin (\theta)$ and $b(x, y)=x \sin (\theta)+y \cos (\theta)$, the image is to be rotated clockwise $\theta$ degrees about its center.

$$
\left(\begin{array}{l}
a(x, y) \\
b(x, y) \\
1
\end{array}\right)=\left(\begin{array}{ccc}
\cos (\theta) & -\sin (\theta) & 0 \\
\sin (\theta) & \cos (\theta) & 0 \\
0 & 0 & 1
\end{array}\right)\left(\begin{array}{l}
x \\
y \\
1
\end{array}\right)
$$

\section{E Waveform}

If the waveform transform is level, then we makes

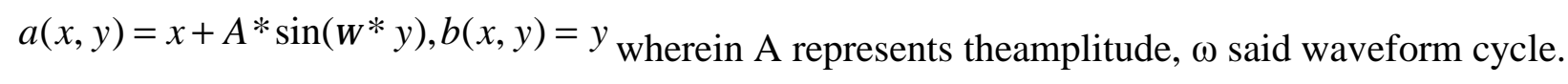

$$
\left(\begin{array}{l}
a(x, y) \\
b(x, y) \\
1
\end{array}\right)=\left(\begin{array}{ccc}
1 & A^{*} \sin \left(\omega^{*} y\right) / y & 0 \\
0 & 1 & 0 \\
0 & 0 & 1
\end{array}\right)\left(\begin{array}{l}
x \\
y \\
1
\end{array}\right)
$$

But if it is a vertical waveform transform, then 


$$
\left(\begin{array}{l}
a(x, y) \\
b(x, y) \\
1
\end{array}\right)=\left(\begin{array}{ccc}
0 & 1 & 0 \\
1 & A * \sin (\omega * x) / x & 0 \\
0 & 0 & 1
\end{array}\right)\left(\begin{array}{l}
x \\
y \\
1
\end{array}\right)
$$

\section{F Vortex}

If we set the coordinates of the vortex points for the $(x, y)$, the scroll angle for the $\theta$, it will be :

$$
\begin{aligned}
& a(x, y)=x^{*} \cos \theta-y^{*} \sin \theta+x 0 *(1-\cos \theta)+y 0 * \sin \theta \\
& b(x, y)=x^{*} \sin \theta+y^{*} \cos \theta+y 0 *(1-\cos \theta)-x 0 * \sin \theta
\end{aligned}
$$

\section{Gray Interpolation Algorithm}

The second requirement of the Geometric transformation of is to run the gray interpolation algorithm. In the input image $\mathrm{f}(\mathrm{x}, \mathrm{y})$, gray value is defined only at integer positions $(\mathrm{x}, \mathrm{y})$. However, in equation (1), the gray value of $\mathrm{G}$ ( $\mathrm{x}, \mathrm{y}$ ) determine by the value of the non integer coordinates $\mathrm{F}(\mathrm{x}, \mathrm{y})$ in general .Therefore, if the geometrical arithmetic as a mapping from $\mathrm{F}$ to $\mathrm{g}$, a pixel in $\mathrm{F}$ will be mapped to the locations between several pixels, the converse is also true. In order to discussion convenient, we specified all pixels are located just at the integer coordinates of the sampling grid.

Commonly used two kinds of interpolation, one is the nearest neighbor interpolation, the other is the bilinear interpolation. Bilinear interpolation can produce more satisfactory results compared with the nearest neighbor interpolation, therefore, we use the bilinear interpolation method in this paper .The principle of bilinear interpolation to the following [2]:

Let $\mathrm{f}(\mathrm{x}, \mathrm{y})$ as a function of two variables, the value of the vertex in the unit square is known, and there have a linear equation:

$$
f(x, y)=a x+b y+c x y+d
$$

We can define a hyperbolic parabolic and four known points have been fitted by the bilinear equation. The four coefficients from a to D must be selected by the $f(x, y)$ of the four apexes. Firstly, it will calculate two above apexes with linear interpolation, we can get:

$$
f(x, 0)=f(0,0)+x[f(1,0)-f(0,0)]
$$

By the same steps, we calculate two down apexes with linear interpolation, we can get:

$$
f(x, 1)=f(0,1)+x[f(1,1)-f(0,1)]
$$

At last, we calculate vertical direction with linear interpolation:

$$
f(x, y)=f(x, 0)+y[f(x, 0)-f(x, 0)]
$$

\section{Experimental Verification}

Now we list the atomic operation key code of the level waveform as an example:

\{

widthAfterS=(int)(curCell.pCell->GetWidth()+2*fabs(A)+0.5);

heightAfterS=curCell.pCell->GetHeight(); 


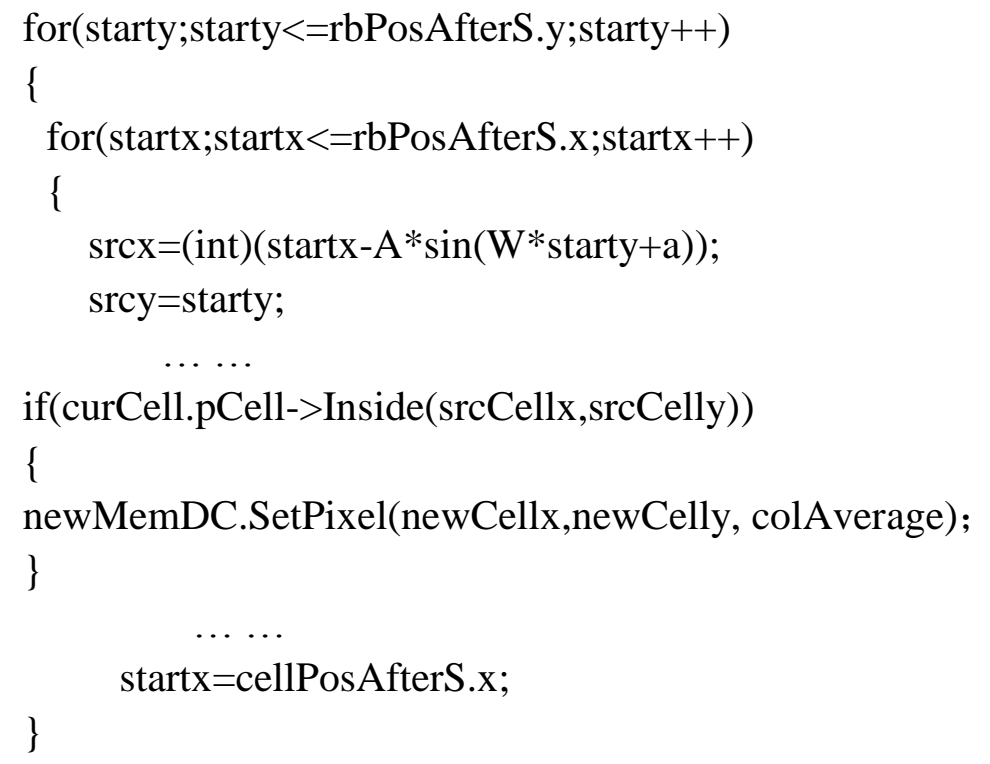

Figure 1 is the two sides successive pattern pattern obtained by two horizontal waveform effect, figure 2 is a suitable pattern after two times the effect of vortex transform.

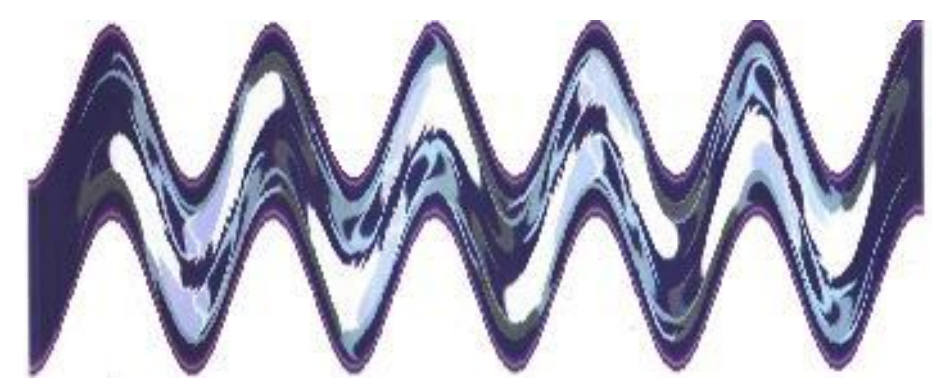

Fig. 1 horizontal waveform transformation

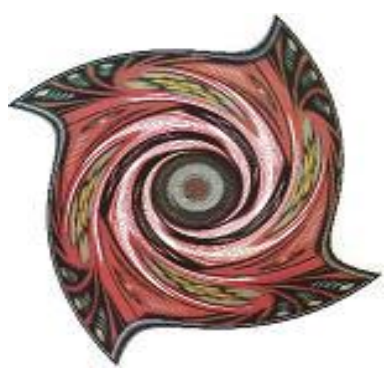

Fig. 2 swirl transformation

\section{Conclusion}

This paper analyzes the six main algorithms of pattern geometry transformation in translation, scaling, rotation, mirror, waveform transform and vortex transform, and analysis and interpolation algorithm of gray value, and then through the experiment validated the ceramic pattern, the results show that the design of ceramic pattern can use geometric transformation algorithm widely, so as to facilitate the rapid building ceramic pattern.

\section{References}

[1] Yang Shuying. VC++ image processing program design. Beijing Northern Jiaotong University press. 2003:79 85.

[2] Liu Xiaozhou, Tu defense. Application of Wavelet bilinear interpolation in optical remote sensing image. Journal of Graduate University of Chinese Academy of Sciences. 2003.20 (1):39 42.

[3] Li S.Tan Y.L. One rapid segmentation algorithm for quasi-circular fruits. Recent advances in computer science and information engineering. 2012, 128: 287-293.

[4] Mesas-Carrascosa F.J., Castillejo-Gonzalez I.L., Orden M.S.Dl.et al. Real-time mobile phone application to supper land policy. Computers and electronics in agriculture. 2012, 85(2012): 109-111. 


\title{
Research on the present development status of micro-nano manufacturing in Jiangsu
}

\author{
Lu Zaigui ${ }^{1, a}$, Liu Ye ${ }^{2, b}$ \\ ${ }^{1}$ Economic and Information Commission of Jiangsu Province \\ 2 Information Eng2ineering College of Yangzhou University \\ aemail, b1424429761@qq, cemail
}

Keywords: micro-nano manufacturing

Abstract. This paper introduces thel distribution, development status and trend of micro-nano manufacturing, the main technical characteristics ,the domestic and international status of the core technology, as well as the advantages and disadvantages of industrialization and so on in Jiangsu. The countermeasures and suggestions for the general development of micro-nano manufacturing in Jiangsu are presented through the enterprise investigation, and the development of the micro nano manufacturing in Jiangsu is summarized and prospected.

\section{Introduction}

The national long-term science and technology strategic development planning study pointed out that the" nano technology and micro system "is the future strategy in the field of high technology and an important direction of Industrialization of high and new technology, is the most potential for development of high technology in the 21st century. China manufacturing 2025 Jiangsu action plan will include "micro-nano manufacturing" in the field of international forward-looking extreme manufacturing technology and equipment. Owing to the distinctive characteristics of the micro scale, mass, low cost, Micro-nano devices and systems play a great role in promoting the modern production and life, for traditional industry upgrading to achieve leapfrog development opportunities and provides the opportunity for the development of the related traditional industrial upgrading it also spawned a number of emerging industries and has become one of the fastest growing industries in the world. widely used in the automotive, petrochemical, telecommunications and other industries, it expanded rapidly to the environment and security, medical and health fields at present, and in the new energy equipment, semiconductor lighting engineering, flexible electronics, optoelectronics and other aspects it also has important application prospect. With the application of micro-nano technology continues to expand, an opportunity for the development of related traditional industries to upgrade is provided, and it spawned a number of emerging industries so that it has been one of the fastest growing industries in the world.

With micro-nano manufacturing problems of basic science research continued to deepen, the scale involved expaned from macro to meso, micro and nano , as well as parameters developing from conventional to unusual or extreme, and it develops from macroscopic and microcosmic to micron and nano scale transition and mutual coupling with structure dimension from $2 \mathrm{D}$ to $3 \mathrm{D}$. manufacturing process and object relates to nano / micro / macro cross scale, scale and interface / surface effects accounted for a leading role. Micro nano manufacturing involves multiple disciplines such as light, machine, electricity, magnetism, biology and so on and a comprehensive study of multi-media field and multi field coupling is needed. As micro-nano devices tend to be smaller scale, higher efficiency and the diversity of materials, the machinability, measurement and characterization of materials have become the key issues. Overall, the trend of the micro-nano manufacturing technology development has many characteristics. its manufacturing scale is more small, its theory is richer, and manufacturing technology is more integrated as well as more discipline overlapping seepage ,furthermore, its application fields is more widely.

The main characteristics of the development of micro-nano manufacturing in China: (1) Basic research results is highlight .The team of micro nano science and technology research center of Jiangsu University took the lead in the country to carry out the basic research of micro mechanical 
design, achieved with the international leading level research, greatly promoted the development of micro machinery research in China, led by professor Ding Jianning in Jiangsu and the colleague experts think "it could be state of art in our country and expand new areas "[1,2] (2) it has achieved important theoretical breakthrough State Key Laboratory of tribology in Tsinghua University carried out fruitful work, explained the causes of frictional interface atoms unstable beating theoretically as well as conditions and the energy dissipation mechanism and developed chip micro friction tester. The Key Laboratory of design and manufacture of micro nano Bio Medical Devices in Jiangsu, led by Yi Hong, developed micro and nano medical device products with independent intellectual property rights and breaking the monopoly of foreign products. (3) Manufacturing technology is international leading. In the field of micro-nano operation, assembly and packaging technology, it has achieved a series of breakthroughs and the integration of optical, mechanical, electrical, biological, chemical and other complex micro and nano systems. Led by academician Zhu Di ,"electrochemical fabrication technology "has made a number of international advanced level of research results in high performance electroforming technology, precision electrochemical machining, micro and nano electrochemical machining etc. (4) Scientific research is rapidly increasing. A lot of work has been carried out in the mechanical characteristic test of micro component by Institute of mechanics of Chinese Academy of Sciences, Xi'an Jiao Tong University, Shanghai Institute of micro system and information technology, North University of China, Tianjin University, and Dalian University of technology.[3-8](5) it involved in a broad area. Micro-nano devices and systems have an important application field of industrial and production, medical and health, environment and safety industry ,involved in the field of science and people's livelihood, Micro-nano friction, wear mechanism and friction reduction strategy, batch, high performance and combination with nano and bio technology is the focus and the leading edge of the development of micro-nano devices and systems[9-13] .Microfluidic devices are an important branch of the micro-nano system, when the fluid channel is small to a certain degree, the mechanism and the external performance have many differences with the macro fluid. At present, micro fluid has become a hot research topic in the basic research[14-18].

\section{development status of micro-nano manufacturing in Jiangsu}

Jiangsu is an important province of Wiener manufacturing industry with complete product range. it formed a relatively complete industrial system, mainly including: MEMS products design and manufacturing, Manufacturing and system development of micro and nano processing equipment , Parts manufacturing in semiconductor processing equipment, related components manufacturing of micro and nano electronic devices, processing of micron powders .

MEMS device product design and manufacturing

In the "Tenth Five Year" period ,MEMS was officially included in the 863 major projects, furthermore, benefit from Education revitalization plan of the Ministry of education, the Chinese Academy of sciences knowledge innovation system, the Fund Committee and the Ministry of science and technology new projects as well as local and corporate investment, its total funding is approximately 5 million RMB. China has made considerable progress in the research and development of MEMS, many achievements have been carried out industrial applications, signs are as follows: micro inertial measurement unit and unit device obtained military applications; and a large number of micro sensor is used as the Shenzhou series of spacecraft control system; a variety of sensing element is for civil products; biological chip, blood biochemical detection system and smart pills and intelligent endoscope mirror started to be used in clinical practice. According to incomplete statistics, there are more than 120 units in the country to carry out research and development of MEMS[19].

Jiangsu is an important base for traditional microelectronics industry, with good conditions for the development of the MEMS industry and a plurality of integrated circuit production line. Some enterprises have international competitiveness in high-end products, pressure sensor, acceleration sensor, and has strong ability of independent research and development. In many sensor production companies, Nanjing Gao Hua Polytron Technologies limited liability company, Kunshan Yu Bin 
electronic technology limited liability company, Chengdu Hong Sheng technology limited liability company, and some scattered manufacturers develop better in the high precision sensor . As a high precision sensor, its main technical indicators are often harsh and complex. the same type of sensor also depends on the specific circumstances For high precision accelerometer, in addition to the Nanjing high technology, there are very few domestic enterprises specialized in the research and production; and for high precision pressure sensor, Kunshan Yu Bin electronic technology limited liability company begins to take shape based on the main technical indexes and foreign similar initial scale.

Ultra compact single chip thermal type (Thermo) acceleration sensor ,made by MEMSIC semiconductor (Wuxi) limited liability company based on standard CMOS technology, use standard CMOS integrated circuit technology of single chip integrated MEMS and ASIC circuit with small volume, light weight, low cost and batch production.It is widely used in mobile consumer electronics products (such as mobile phones) and high-end sensor, such as automotive electronics, industrial applications. MEMSIC triaxial single chip MEMS magnetic sensor has a $100 \%$ independent intellectual property rights, using the technology of MEMS and CMOS standard scale integrated circuit, with the advantages of small volume, light weight, low cost and batch production, and can integrate with IC system or extended integration of other MEMS sensors .The sensor of the technical indicators reached the international advanced level, which fills the gaps in this field in China, and will play a positive role in supporting the national MEMS industry and the corresponding automotive and consumer electronics industries development. MEMSIC semiconductor single chip magnetic sensor is mainly used in many fields of aerospace, automotive, industrial, consumer etc.. Automotive, consumer electronics and mobile phones accounted for about $70 \%$ of the market share of magnetic sensing applications. Nanjing Gao Hua Polytron Technologies limited liability company is to develop high reliable MEMS sensor and sensor network engineering , as a national high-tech enterprise, which launched sensor (Union) network industry alliance in Jiangsu. It is Nanjing MEMS sensor Engineering Technology Research Center and Jiangsu province enterprise graduate student work station. Its typical products include capacitive micro accelerometer, wafer level vacuum packaging of high precision capacitance type pressure sensor. In particular, the sensor that is made by the independent research and development performances stability in the range of -50 -125 with anti-interference and good stability (within 30 days), breaking the technical embargo of the developed countries. Due to historical reasons, Famous companies from the United States and Europe occupy the top in miniature and high frequency dynamic pressure sensor and other high-end products. although the high-end manufacturing itself is not a mass product ,enterprise scale is not large. Because of its importance, it is important to the promotion of military equipment, high-end ritual significant. Micro pressure sensor of the army enterprise is the United States Kulit (kulite) company. the minimum size of the product is $1.7 \mathrm{~mm}$ in diameter and the thinest product is $0.64 \mathrm{~mm}$. Shuangqiao sensor company's minimum product is $2 \mathrm{~mm}$ in diameter, the thinnest is $1 \mathrm{~mm}$. It basically reached the international advanced level, which make a contribution for our country. however, there is a certain gap in the performance and variety of complete between products and it need to work hard. Right now, for example, in high dynamic measurement of the sensor mainly monopolist is Kistler and PCB. Table 2.1 is the comparison of Shuangqiao sensor company and the two companies. It can be seen that the company is obviously superior to the key parameters in the natural frequency and the rise time, and its high dynamic key technical indicators have reached the international leading level.

Table 2.1 comparison of the product performance index of Kunshan Shuangqiao sensor company and Kistler company and PCB

\begin{tabular}{clcc}
\hline unit & \multicolumn{1}{c}{ Shuangqiao sensor } & Kistler & PCB \\
\hline \multirow{4}{*}{ key } & \multicolumn{1}{c}{$\begin{array}{l}\text { Semiconductor silicon } \\
\text { pressure resistance } \\
\text { principle and fully flush } \\
\text { structure with flat back } \\
\text { pressure on the back } \\
\text { natural }\end{array}$} & $\begin{array}{c}\text { Piezoelectric quartz } \\
\text { and Flush package }\end{array}$ & $\begin{array}{l}\text { Piezoelectric } \\
\text { piezoelectric or flush } \\
\text { package }\end{array}$ \\
\hline
\end{tabular}




\begin{tabular}{|c|c|c|c|c|c|}
\hline \multicolumn{6}{|l|}{ frequency } \\
\hline $\begin{array}{l}\text { corresponding } \\
\text { time }\end{array}$ & $0.1 \mu \mathrm{s} \sim 1 \mu \mathrm{s}$ & $4 \mu \mathrm{s}$ & & $2 \mu \mathrm{s} \sim 4 \mu \mathrm{s}$ & \\
\hline $\begin{array}{l}\text { Available } \\
\text { bandwidth }\end{array}$ & $1 / 3$ Natural frequency & $\begin{array}{c}1 / 10 \\
\text { frequency }\end{array}$ & natural & $\begin{array}{l}1 / 10 \\
\text { frequency }\end{array}$ & Natural \\
\hline $\begin{array}{l}\quad \text { Anti strong } \\
\text { infrared } \\
\text { interference }\end{array}$ & $\begin{array}{lr}\text { gold } & \text { diffusion } \\
\text { composite } & \text { machine } \\
\text { sputtering metal } & \text { screen } \\
\text { and shield design }\end{array}$ & no & & no & \\
\hline
\end{tabular}

micro-nano processing equipment manufacturing and system research and development

Jiangsu micro-nano processing equipment manufacturing and industries related to the system are mainly concentrated in the industrial park in Suzhou .Suzhou Institute of nano technology and nano bionics of Chinese Academy of Sciences (hereinafter referred to as Suzhou Institute of nano) achieved a seamless transition from the microelectronic technology to nano electronic technology; it used high precision nanomaterials and technology means to improve the traditional microelectronic products manufacturing process and the performance of integrated circuit products, especially the reliability of microelectronic products. Suzhou new nano crystal photoelectric limited company is the first to have a complete industrial chain of enterprise innovation ,from graphical to substrate to extension to chip to package to lamp assembly, in the control of InGaN Quantum Dots growth and LED efficiency droop with high power, LED heat radiating mechanism design, it made a number of research results . the maximum temperature of the chip packaging process must be lower than $280^{\circ} \mathrm{C}$, less than $10 \mathrm{~s}$ and main wavelength measurement error must be $+0.5 \mathrm{~nm}$.

Suzhou Navier Technology limited company put attention to the growth and development of nitride materials with high quality and large size, provide all kinds of Gan materials for Industry and research institutions and is first gallium nitride substrate chip suppliers in China. Suzhou Diener precision equipment limited company is mainly engaged in MEMS sensor packaging and testing equipment research and development and can provide customized packaging and testing automation system design for enterprises. it provide complete solutions to realize the batch manufacturing. For example, tensile testing equipment of independent research and development is specifically designed to test various lead strength test equipment and can adapt to the PCB board, dual in-line tube shell, dip, cob and other packaging structure of wire operation. Its positioning accuracy is $5 \mu \mathrm{m}$, visual recognition accuracy is $10 \mu \mathrm{m}$, and precision measuring degree can reach $0.001 \mathrm{~N}$

As a new field, Nano imprint lithography equipment's domestic development is relatively slow and the domestic nano imprint lithography equipment and supplies often relied on import. In recent years, although there are some companies providing such products, more being direct introduction of foreign equipment, not introducing key technologies, and enterprises with independent intellectual property rights is even more rare. Suzhou optical rudder micro-nano technology limited company is mainly engaged in nano imprint lithography equipment, nano imprint template and nano imprint lithography, and provide nano imprint lithography and micro nano processing solutions. The nanoimprint equipment mainly has four kinds: micro contact type nano imprint equipment, thermal and UV nanoimprint equipment, UV flexible die nano imprinting apparatus and hot embossing and UV imprint various nano imprinting apparatus alignment; the nano imprint template includes large area nano line shaped template, large area of nanopores and columnar template and nano imprint template; the nano imprint lithography glue products are mainly for nil series nano imprint consumable products. Its products' $\mathrm{R} \& \mathrm{D}$ and production is mainly facing to the customer .As an example, its large area of nanopores and columnar template's area is $1 \sim 10 \mathrm{~cm}^{2}$ and size resolution can reach the minimum $30 \mathrm{~nm}$. The minimum sample period of porous structure is $70 \mathrm{~nm}$, the highest aspect ratio is 3 and minimum sample period of the columnar structure is $100 \mathrm{~nm}$.

parts manufacturing in semiconductor processing equipment

International semiconductor equipment's global procurement need is about \$200 billion, mainly came from chip manufacturers and vendors supporting in Asia, South Korea, China Taiwan, Japan, mainland China, Singapore and the United States.And these devices, especially high-end chip 
production depends on some advanced equipment and production technology, which is on the control of the United States and Japan and other western developed countries. With the growing demand for high-end semiconductor products in China, the development of high-end semiconductor equipment has become a the urgent task on national security and market development .

At present, the leading maker of the large scale integrated circuit etching machine is the United States AMAT company, Japan TEL company and the United States LAM company. AMAT and TEL2013 were integrated and the combined AMAT-TEL chip equipment sales reached $\$ 32$ billion, accounting for the 1/4 total global output of the chip. Chinese Micro company has built a capacity of one billion U.S. dollars and the next five years is expected to produce 100 units and sales of 2-3 billion.

Pioneer Semiconductor Technology limited company to achieve a set of precision machining and surface treatment as one of the semiconductor micro nano processing equipment parts manufacturing .The development work is in the groundbreaking in semiconductor processing chamber $(14 \mathrm{~nm}$ process chamber), cavity heater and electrostatic chuck ( 8 inch) in China.Its semiconductor processing equipment parts in the semiconductor equipment limited company, North Microelectronics limited company is the best in the world's global component procurement projects.Pioneer $\mathrm{Co}$ is in the top four in the global procurement among 350 micro Semiconductor Equipment Co., Ltd., the second of the supply of North Microelectronics Co., Ltd. Taiwan exports of equipment parts

Except for the Shanghai Songjiang, Foxconn, pioneer Semiconductor Technology Co., Ltd is only the only company similar with international standards of large-scale integrated circuit plasma etch chamber in technology in our country, as a professional manufacturer of integrated processing and table processing.Project products have a good cooperation agreement with domestic large customers (micro Semiconductor Equipment Co., Ltd., North Microelectronics Co., Ltd.) with the product of corrosion resistance, high breakdown voltage, reliable quality and excellent performance. In particular, the orrosion resistance performance the surface treatment part of the component is high, whose thickness of the oxide layer is uniform. The service life of the product is prolonged, and the cost of the user is saved. Pioneer Semiconductor Technology Co., Ltd. has reached a very high level, enough to comparable to similar foreign advanced technology in the processing of semiconductor equipment parts manufacturing. It is in the domestic leading position ,whose key technology is surface treatment technology, extrusion honing micro manufacturing process,.

micro- nano devices manufacturing

At present, the domestic production of the mask is mainly based on etching technology. although this technology is more mature, restricted by the import material, so the special production of domestic mask has long stalled. Life in common mask is only made by some metal products company, such as Beijing Dongfang Huawei Technology Co., Ltd., Shenzhen Hong Yesheng metal product Limited company, etc., whose production level has reached the top level of ordinary metal manufacturing industry but still not high-end.At present, in the metal etching processing industry, the development of Shenzhen beaconton Electronic Co., Ltd is in a mature.. the minimum stable processing microporous can reach $0.1 \mathrm{~mm}$ and minimum thickness of the product can reach $0.03 \mathrm{~mm}$, the highest precision can reach $+7.5 \mathrm{~m}$. It has been close to the highest level of etching technology,.

The main products of Kunshan, Jiangsu Yun Sheng Ji Photoelectric Technology Co., Ltd. are high precision metal mask template for OLED deposition, metal mesh plate for photovoltaic solar screen silver paste printed .Its technological achievements originate from the National 863 project named " the development of high precision steaming plating mask plate". at present, the company has completed the research of the steaming plating mask for the PMOLED, and have the production capacity, possessing strong technological foundation to undertake the development of AMOLED.The core technology is the electroforming technology with advantages of high precision and arbitrary thickness (more than traditional $0.04 \mathrm{~mm}$ ), trapezoidal natural taper, smooth hole wall, high precision, more high aperture ratio than an etching reduction technology can realize products and other .Table 2.2 is the comparison of main technological parameters of Yun Kunshan Sheng Ji products and 
international manufacturers to find that the manufacturing precision and the tension and the network sag has reached the international advanced level

Compared with domestic counterparts, OLED first project production line of Anhui Dafu Photoelectric Technology Co. Ltd. has been officially put into operation. It is understood that Dafu heavy industries, Dafu photoelectric project are invested and built by Anhui Dafu Heavy Industry Technology Co., Ltd. with 15 million yuan, whose main construction contents include 3 production line of metal mask plate membrane of active organic light emitting display (AMOLED) evaporation, annual production capacity of super fine metal mask plate membrane being expected to reach 6000 sets, flexible devices (wearable electronics) 500 million pieces, precision electronic hardware components 5000 million, an annual output of 1000 sets of intelligent industrial robots, and annual output of mobile communication base station antenna reaching 70 million. Shanghai Hui and photoelectric Co., Ltd. is a high-tech company specializing in the production of small and medium size AMOLED display and the next generation of display technology. The company is currently the first domestic and most perfect and the most advanced 4.5 generation low-temperature polysilicon (LTPS) AMOLED production line, with large-scale production capacity

Table 2.2 comparison of main technical parameters of metal mask for OLED evaporation between Kunshan and international manufacturer

\begin{tabular}{|c|c|c|c|c|c|}
\hline Index & DNP & TOPPON & & $\begin{array}{c}\text { Allow } \\
\text { appreciation }\end{array}$ & \\
\hline Panel size & $450 \mathrm{~mm} \mathrm{X} \mathrm{550mm}$ & $\begin{array}{l}450 \mathrm{~mm} \\
550 \mathrm{~mm}\end{array}$ & $\mathrm{X}$ & $\begin{array}{c}450 \mathrm{~mm} \\
550 \mathrm{~mm}\end{array}$ & $\mathrm{X}$ \\
\hline $\begin{array}{l}\text { Open } \\
\text { position } \\
\text { precision }\end{array}$ & $\pm 5 \mu \mathrm{m}$ & $\pm 5 \mu \mathrm{m}$ & & $\pm 5 \mu \mathrm{m}$ & \\
\hline $\begin{array}{l}\text { Open } \\
\text { position } \\
\text { precision }\end{array}$ & $\pm 3 \mu \mathrm{m}$ & $\pm 4 \mu \mathrm{m}$ & & $\pm 3 \mu \mathrm{m}$ & \\
\hline $\begin{array}{r}\text { Stretch } \\
\text { mesh sag }\end{array}$ & $\leq 120 \mu \mathrm{m}$ & $\leq 150 \mu \mathrm{m}$ & & $\leq 100 \mu \mathrm{m}$ & \\
\hline
\end{tabular}

In the share of the AMOLED market, Samsung is undoubtedly the industry's leading enterprises with the advantage of Accounting for 9 of the global market share In fact, Samsung lead the AMOLED market boom until 2009 GALAXY phone were hot. As a result of the existing LCD in the former, although the initial development of OLED is not very smooth, PMOLED (passive matrix OLED) is mainly used for mobile phone, automotive, industrial applications and other markets; And at present, the world has more than 100 research institutes and enterprises to invest in the development and production of OLED, including the global industry giants, such as Samsung, LG, SONY and other companies.

micron powder materials processing

Micron powder material is mainly used for conventional powder metallurgy and injection molding, iron core, diamond tools, diamond catalyst, nutrition iron supplementation, microwave absorbing materials.

Among them, micron carbonyl iron powder is widely used in powder metallurgy, metal powder injection molding, powdered catalyst of synthetic diamond, diamond tools and hard alloy product of binder, high frequency and high quality factor, high permeability, low power soft magnetic materials, microwave absorbing camouflage material, electromagnetic compatible devices, $\mathrm{Mr}$ (magnetic damping), nondestructive flaw detection, magnetic inks, iron deficiency for the crowd of iron supplement etc..

The main parameters and characteristics of micron carbonyl iron powder made by Jiangsu superfine metal powder Co., Ltd. includes that the average particle size is 3-3.5um and oxygen content is less than or equal to $0.2 \%$, carbon content is less than or equal to $0.7 \%$.It has high purity, does not contain harmful heavy metals and contain only a small amount of $\mathrm{C}, \mathrm{N}$, O ; Except for fine 
particle size and narrow distribution, good sintering activity; powder into spherical and good fluidity, its accumulation slope is small and particle profile is concentric onion like layered structure etc..The company achieved the advantage of the synthesis of short cycle, high yield, the pyrolysis of intelligent, controllable, the formation of independent intellectual property rights of the process and equipment system. Its production scale is the country's largest, with the domestic leading technology level.

Only three company ,including BASF in Germany, the SINTEZ in Russia, the ISP in United States, product carbonyl iron powder on the international and its technical level is basically equivalent as well as product application surface.Because the product is widely used in national defense and high-tech industry, its production technology, equipment, scale have not been reported .Only some scattered and vague propaganda reports, and sporadic and simple product promotional materials printed by domestic agents published for civilian can be retrieved.The development and production of carbonyl iron powder in China has a history of nearly 40 years, but it has not been in a scale with backward technology and equipment, low production, unstable quality and narrow application, mostly relying on imports. Jilin Jien nickel industry is domestic listed companies. It is more speculation and the product quality is not stable. 857 original nuclear industry factory, Shaanxi Xingping chemical fertilizer plant has small quantity production, as two Western military industrial enterprises, whose total annual production isless than 200 tons; Guangdong Zhongshan Yue Dragon (Jiangxi Yue is a company with less than 300 tons annual production .Because of the system and the location, for decades, the process is small and obsolete with no technological progress. The production is of small scale, long reaction cycle, low yield and low quality and stability, etc

\section{conclusions and suggestions}

Overall, relative to the developed countries ,micro nano manufacturing industry is in the primary stage in Jiangsu. micro nano manufacturing equipment is mainly imported, lacking of talents for research and development and equipment input.Lacking of enterprise, industry and national standards, the industry chain is not complete, especially the lack of downstream industries, resulting in micro nano manufacturing low demanding for the quality of related products, so as to make the industry chain lack economic benefits leading to the loss of personnel, equipment and capital orientation. The talent strength of the enterprise is not strong, even no research and development department. Mainly by individual combat mode, it lacks research cooperation with universities and Research Institute; part enterprise of a certain innovation ability is too small and its degree of automation is not high. In view of the present situation of micro nano manufacturing industry in Jiangsu, the following suggestions are put forward:

(1) In the point of view of the micro nano manufacturing industrial structure, micro nano manufacturing industry design and manufacture of MEMS device as the main body with a certain industrial base in Jiangsu. Part of the products fill the domestic blank and performance of a small number of products is in the international leading level with higher share in the domestic product. On the basis of this, suggestions that it is necessary to increase MEMS device design, manufacturing and $\mathrm{R} \& \mathrm{D}$ equipment, fund and personnel input are given to make MEMS design and manufacturing industry in Jiangsu become the domestic first-class, and international advanced level characteristic industry industry, driving a whole the development of micro nano manufacturing industry. In addition, it is recommended to increase the micro nano manufacturing equipment and the R \& D investment of related parts and components, improving domestic equipment market share of micro-nano manufacturing, in order to reduce the cost of micro nano manufacturing, as strong support for a whole micro nano manufacturing industry in our province and even in our country.

(2) It is necessary to increase the investment of micro nano manufacturing industry fund project and guide micro nano devices and systems research and development boom, to achieve ways to solve key technical problems and to increase industry and national standards of the micro nano manufacturing related products.Due to the late start and the not mature technology and lacking the investment of capital and talent, Domestic micro nano devices has still a certain gap in terms of 
performance and foreign large quantities of automation products. Therefore, we should start from the fund project investment, attracting research personnel into research and development and technical research, accelerate the transformation of research results, increase the standards of enterprise, industry or national, in order to enhance the competitiveness and credibility of products.

(3) It is suitable that downstream industry demand promote the development of micro nano manufacturing industry chain. Current international products related to micro-nano technology mainly includes automotive electronics and consumer electronics products. the electronic products consumption is dominated by imports. imports of micro nano devices and system has excellent performance but expensive and domestic micro nano technology products are cheaper. in line with the industry standard, to improve the competitiveness of domestic micro nano devices product and expand the product demand could give the enterprise innovation to rise profits .

(4)It is necessary to input into the construction of semiconductor processing test center. Micro nano processing equipment, in the high-end of Micro nano manufacturing industry, in the short term is difficult to achieve complete localization and industrialization, and semiconductor processing and testing process of micro nano manufacturing process has an important role in micro and nano device design, system and equipment research and development, micro -nano manufacturing key technology research and even become a part of the business development constraints. Therefore, it is of great significance to set up the semiconductor processing test center, to realize the sharing of equipment resources, and to the development of micro nano manufacturing industry in our province and even in the whole country.

\section{References}

[1] Ding Jianning, Meng Yonggang, Wen Shizhu. Size effect of Polycrystalline silicon micro cantilever beam fracture failure strength [J]. China Mechanical Engineering, 2001, 12 (11): 1228 1231

[2] DING Jianning, MENG Yonggang, WEN Shizhu. Specimen size effect on mechanical properties of polysilicon microcantilever beams by deflection using nanoindentation. Materials Science and Engineering B., 2001, 83(1-3): 42-47.

[3] Mei niansong, Huang Qing'an the online test of MEMS film transverse rupture strength--a thermal driving method [J]. Micronanoelectronic technology, 2003 (7 / 8): Sweden

[4] Wu Hao, Yu Yonggang, Cai Jun Su,etc. test method of Fatigue property of polysilicon thin film [J]. Harbin Industrial University Journal, 2006, 38 (4): 592-595.

[5] Denggang. Measurement of Micro material mechanical property [D]. Shanghai: Shanghai Institute of Microsystem and information technology, 2003

[6] Jiang Zhuangde, Wang Hairong, Zhao Zexiang, et al. Micro mechanical mechanical properties tester: China, 00226180 2000-12-29. [P].

[7] Zhang Taihua, Yang Yemin, Zhao Yapu, et al. Progress in mechanical properties of.MEMS materials, [J]., 2002, 32, (4): 545-562.

[8] Su Caidiao, Wu Hao, Guo Zhanshe, et al. Test system of Mechanical properties of single crystal silicon micro component [J]. mechanical strength, 2005, 27 (4): 456-459.

[9]Li Zhijun, , Leng Yongsheng, Zou Kun, et. experimental study of surface force apparatus and solid surface micro contact mechanism [J]. Tribology, 2000, 20 (5): 336-339.

[10]Zhang Xiangjun of, Meng Yonggang, Wen Shizhu The AFM experimental study of MEMS microcantilever structure deformation rules [J]. Mechanical science and technology, 2005, 24 (8): 966-968.

[11] Shi Hongsheng, Wang Hui, Hu Yuanzhong. Design of elastic cantilever beam friction force measuring mechanism [J]. mechanical science and technology, 2004, 23 (11): 1327-1330.

[12] Tang Zhenan, Li Xin, Xu Jun. Preparation and characterization of diamond like carbon films for micro electro mechanical systems [J]. Journal of materials science, 2004, 18 (6): 582-586

[13] Qing Tao, Shao t m, Wen Shizhu. Study of relative humidity influence on material surface adhesion force [J]. Tribology, 2006, 26 (4): 295-299. 
[14] Wu Jiangang, Yue Ruifeng, Ceng Xuefeng, et al. Micro droplet driving chip based on the principle of electro wetting on the medium [J]. Journal of Tsinghua University, 2006, 46 (7): 1341-1344.

[15] Ding Yingtao, Yao Zhaohui, he Feng. Study on the characteristics of gas flowing in micro nozzle [J]. engineering mechanics, 2004, 21 (3): 190-195

[16] Xie Haibo, Fu Xin, Yang Huayong, et al. Simulation and micro-PIV research on classical micro channel flow [J]. Journal of mechanical engineering, 2006, 42 (5): 32-38.

[17] ZHANG Zhongqiang, YE Hongfei, LIU Zhen., DING Jianning, et al. Carbon nanotube-based charge-controlled speed-regulating nanoclutch. Journal of Applied Physics, 2012, 111: 114304.

[18] ZHANG Zhongqiang, ZHANG Hongwu, ZHENG Yonggang, WANG Lei, and WANG Jingbao. Gas separation by kinked single-walled carbon nanotubes: Molecular dynamics simulations.Physical Review B, 2008, 78: 035439.

[19] Wang Liding, Zhu Jinkui, Liu Chong, Luo Yi. Research progress of micro nano manufacturing in China [J]. Journal of mechanical engineering, 2008, 44 (11): 1-12 


\title{
3D laser scanning technology in the application of modeling in mining subsidence area
}

\author{
Zhao Xiaoping ${ }^{1, a}$, Zhang Jian ${ }^{2, b}$, Liu Wenlong ${ }^{1, c}$ \\ 1.Beijing Polytechnic College, Beijing, China 100042 \\ 2.Remote sensing surveying and Mapping Institute in Henan province Zhengzhou, China 450003 \\ azxpcumtb@126.com, b461910321@qq.com, clwl@bgy.org.cn
}

Keywords: 3D laser scanning technology; mining subsidence; points cloud filtering ; Kriging interpolation method.

\begin{abstract}
By means of 3 d laser scanning technology has rapidity, non-contact, high density, high precision features, such as the mining subsidence area on the station point cloud data registration, denoising, model building, such as pretreatment, can get real and effective information and visualization expression results subsidence area. The research results show that the $3 \mathrm{~d}$ laser scanning technology is applied to the mining subsidence area, to build accurate model of subsidence area, meet the requirements of subsidence analysis, has engineering practical value.

Currently, as pillar energy, coal is mined on a big scale. However, mining brings a lot of environmental and geological problems to diggings, such as subsidence, building damage and harmful substances into the ground water ${ }^{[1]}$. Traditionally, levelling instrument and total station are used to monitor the subsidence in diggings. With the development of surveying technology, many new techniques are also applied. Sheng Yehua et al. acquired digital image pairs of subsidence area by high resolution digital camera, built DTL algorithmic model on the basis of image control points set on site, and calculated a series of 3D coordinates which can describe the morphological characters of subsidence area. Under the support of GIS, DTM of initial terrain and status of subsidence area were built. Through the analysis of two-stage DTM, specific morphological characteristic parameters of subsidence area were calculated ${ }^{[6]}$.Shi Xianling, Guo Hongxing et al. set up monitoring network and measured spatial coordinates of stations by GPS-RTK and GPS quasi dynamic measuring. After solving and analyzing the data, they found an law of surface movement with good effect ${ }^{[2-4]}$.

With the help of 3D laser scanning technology, we can effectively get dense point cloud data and large-scale subsidence information. The biggest advantage of this technology is that 3D laser scanning can acquire high-precision point cloud data directly without prism. Under the advantage of fast acquisition of high-precision 3D information, 3D laser scanning technology can collect a large number of dense point cloud data of subsidence area in a short time. And it can build DTM of complex and anomalous scence quickly, response the true appearance of subsidence basin. Moreover, this is the basis of calculating estimated parameters and analyzing ground movement. Compared with artificial measurement, 3D laser scanning technology is competitive and has more broader development space.
\end{abstract}

\section{General situation of study area}

In order to study the scheme of observation station layout of surface movement, we chose a coal mine in Jiaozuo as experimental area. Under the influence of mining, the subsidence area is calathiform and the size is $140 \mathrm{~m} \times 120 \mathrm{~m}$. This area is of wide vision, and there is no big trees and buildings. In this test, we use Leica HDS 4400 3D laser scanner, high density mode is used to collect data. Panoramic scan once requires $12 \mathrm{~min}$. If the effective scanning radius is $80 \mathrm{~m}$, the scanning area is $20096 \mathrm{~m}^{2}$. 


\section{Acquisition of point cloud data}

Acquisition of data with 3D laser scanner can divide into three parts: lay observation stations, scan and scan accurately. In the scanning phase, scanning should be carried out continuously, and the cycle of scanning should be shortened. Scanning plan should be adjusted according to actual situation. If the scanning area is flat and bare, we can increase sampling interval and shorten the scanning time. Conversely, if the terrain is complex or there is many vegetation, the sampling interval should be decreased; when scan the serious occlusion area, it is different to get point cloud data, we should add observation stations. Observation stations are designed on the maximum subsidence main section, both trend and dip section, just like traditional hatching observation stations. In the two segment of observation line, we should ensure that the scanning area must be larger than subsidence area.

\section{Point cloud data preprocessing of subsidence area}

\section{Topographic LiDAR point cloud data registration}

Registering according to points of the same name is a common method to fit point cloud data into unified coordinate system. At first, we put three or more targets in the scanning area. Scan the target area with high density mode and get the point cloud data of scanning area. After scanning the target area, we scan the targets precisely and extract the center point coordinates. The conversion parameters are calculated by using common point coordinate. When register the data among all stations, the error is less than $1 \mathrm{~cm}$. The impression drawing is showed in fig. 1 .

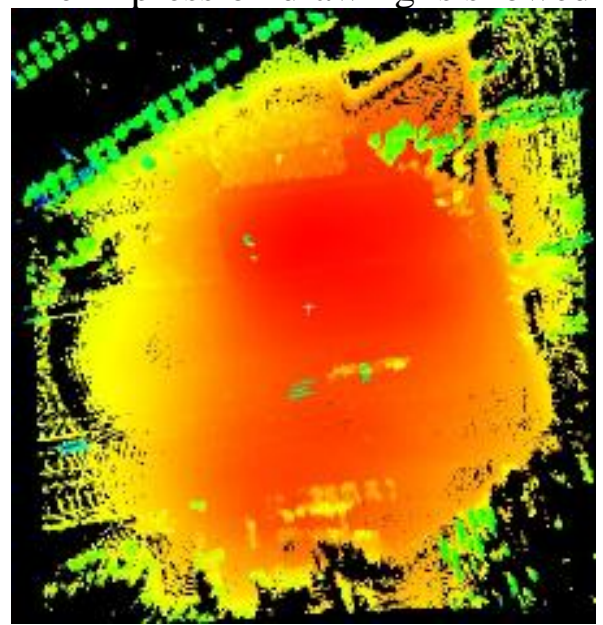

Fig 1. Splicing map of initial point cloud data of subsidence area

\section{Point cloud data denoising and optimization}

point cloud data denoising

The main task of the laser point cloud data pre-processing is how to accurately and effectively remove the chaotic noise data, retaining only the measured region exposed the land surface information. Ground 3D laser scanner in the process of data acquisition, will be recorded to all the objects can be scanned information, such as building(structure), water, vegetation and the land surface information. Different objects have different characteristics, in general, in addition to the land surface of real data, the 3D laser scanning data of subsidence area can be divided into high elevation of the building(structure) data and data of weeds in low elevation.

data optimization

Point cloud data optimization is generally divided into two kinds, removal of redundancy and sparsing simplify. Redundant data refers was given a complete point cloud model, but also generate a large number of overlapping data after multi-station data registration. This overlap of data will take up a lot of resources, reduce operating and storage efficiency, but also affect the efficiency and quality of the modeling. In some non-essential stand point cloud may appear too close, then use sparsing simplified. In the laser point cloud data of subsidence area, there are obvious geometrical features of building(structure), trees and electric poles. The average height of the building(structure) 
is the highest, and the elevation of the ground objects such as trees and poles is between the surface point and the elevation of building points. Therefore, according to the different features of different elevation to remove some noise data.

Data filtering process

According to the principle of filtering and the characteristics of subsidence area, first of all statistical data points within each elevation point cloud distribution range, and draw elevation map. According to the point cloud data elevation distribution set threshold, then remove a portion of higher elevation of the noise, then, after the removal of data in accordance with X-Y plane grid. The study area is divided into $\mathrm{M} \times \mathrm{N}$ regular grid, and then import the split data into software for processing, only preserve the surface data, remove the low vegetation information of the point cloud.

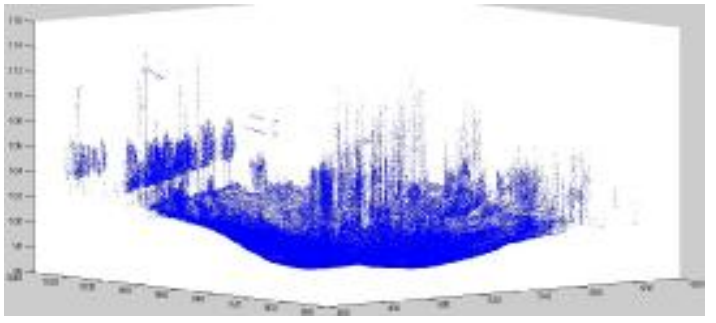

(a) Noise data before removing

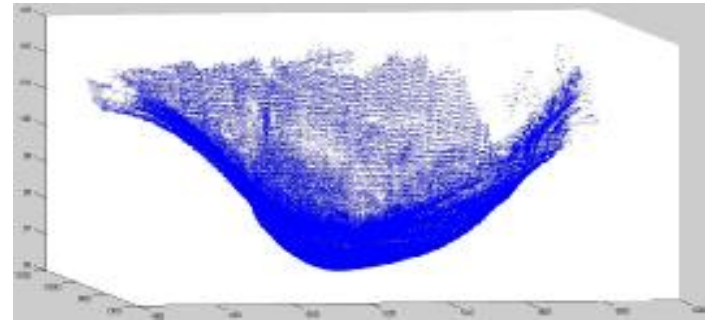

(b)Noise data after removing

Fig.2 Comparison of point cloud before and after denoising

As can be seen from figure 2(a), the subsidence zone is large, rough filtering can not be completely removed some low noise, and it is possible to put a higher surface elevation point mistook the noise data to removed. In order to solve this situation, the situation is complicated areas must be divided into a plurality of fine grids. Filter out the noise within each grid, After the grid division, it can be further removal of these low vegetation in the small grid,as can be seen from figure 2(b).But this method cannot automatically and completely filter the noise of low vegetation, to be further improved.

\section{Build the surface model}

For non-topological relations point cloud data, the model poorly constructed using these scattered points directly. To get a complete point cloud data, and accurate expression of surface subsidence characteristics, needs to use the interpolation method to compensate for the missing data. There is a lot of interpolation, Kriging interpolation method is a commonly used interpolation method based on geostatistics, the method can be used to generate a prediction surface, but also given the accuracy of the results or the measure of certainty. After the data interpolation process, it need to build a grid model of the scanning area, it is not only able to clearly express the basic information of terrain surface, but also preparing for the forecast and analysis of future changes in the terrain surface. According to the characteristics of subsidence area, choose Kriging interpolation to fill the voids, by adjusting the spacing of the required grid, such as figure 3 . According to different elevation, giving different colors and eventually generate terrain surface renderings, such as figure 4 .

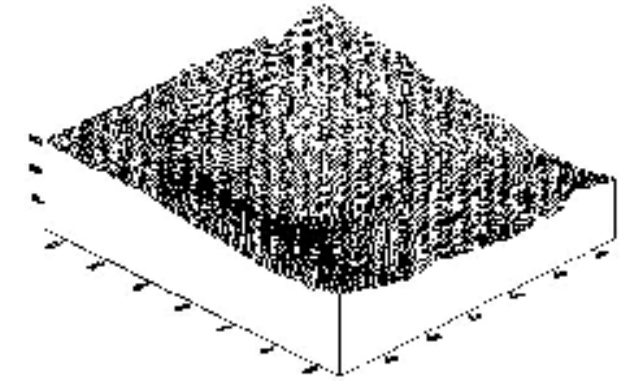

Fig. 3 Grid map

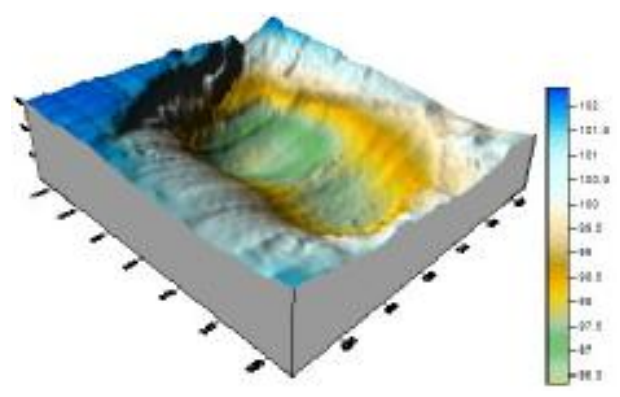

Fig. 4 The surface

rendering model 


\section{Comparison of section line point cloud data}

When using 3D laser scanner to set observation station, it can be set up along the lines ends to higher places. This can not only guarantee the point cloud density in the central area of the subsidence basin, but also can give attention to the accuracy of the point cloud in the edge region of the subsidence basin.

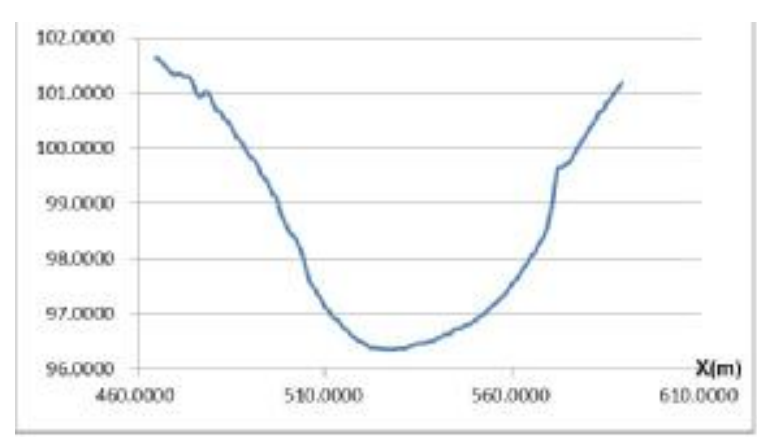

) dip section diagram

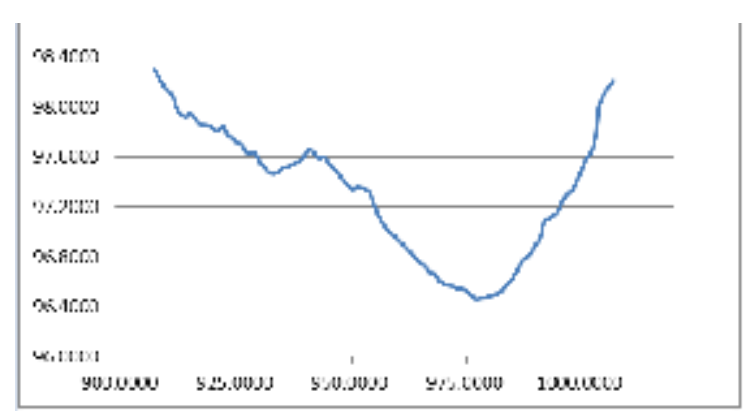

(b) trend section diagram

Figure 5 The subsidence curve profile diagram

The data points collected by 3D laser scanner, can make the seam trend(or dip section) of the analysis of the line based on the 3D modeling, more accurate than the traditional measurement methods to reflect the trend of surface changes. When analyzing the subsidence, as shown in the figure 5 of the sinking value, it can be obtained by the subtraction value of the data before and after deformation.

\section{Concluding remarks}

This paper is on the basis of analyzing the ground 3D laser scanner for mining subsidence monitoring, through the method of stepwise refinement grid, it can effectively remove the point cloud noise, provide basic data for application of subsequent point cloud. For the filtered produce "empty" point cloud data, Kriging interpolation method is used to interpolate the data, obtained better effect surface model. Compared with traditional research methods, 3D laser scanning technology has great advantages in 3D modeling and deformation analysis of mining subsidence area.

\section{References}

[1] Kan Wu, Yunjia Wang, Suiquan Wang, mining subsidence monitoring and forecasting technologies [M], the Chinese environmental science press ,2012.

[2] Xianling Shi, Shubi Zhang. GPS application to dynamic measurement of land surface movement observation station [J]. Western exploration engineering,2006,(12):137.137.

[3] Gang Wang,Guangli Guo,Ling Li. GPS-RTK application of coal mine surveying and mapping and surveying in the mountains [J]. China mining and construction,2011,40 (2): 41.

[4] Hongxing Guo, Yufu Hang, Baoji Zhao. RTK technology and its application in mining subsidence monitoring [J]. Shanxi coal science and technology,2007 (2): 31-33.

[5] Jingjing Li,Zengzhang Guo. Based on D-InSAR technology of mining subsidence in coal mining area [J]. Journal of Henan Polytechnic University (natural science Edition),2006,25 (4): 306-309.

[6] Yan Jiang,Junhai Gao. Synthetic aperture radar interferometry technology application to mining subsidence monitoring [J]. Mine survey,2003 (1): 5-7.

[7] Wensheng Mei, Zhenglu Zhang, Quanyi Zhang. Study on the application of survey robot in deformation monitoring [J]. Dam safety, 2005(5):33-35.

[8] Zhenli Ma, Changdong Ji, Dongfeng Ren. GPS joint operations with the robot in deformation monitoring of open pit applications [j]. Mine surveying, 2007(1):41-42. 
[10] Shuanghong Wang, Bin Wang. Application of survey robot in deformation monitoring [J]. Journal of disaster prevention technology College, 2005 , 7(3):38-42.

Project funding on the market in Beijing, Project No.: KM201610853006.

Author introduction: Zhao Xiaoping(1976-),female, Han, Xinzhou Shanxi Province, associate professor, major in teaching and studying new technology of surveying and mapping. 


\title{
Recycling and Reuse of Sulfur Hexafluoride in Guangdong Power Grid Corporation
}

\author{
Min Chen \\ Guangdong Power Grid Co., Ltd. Yuedian Building, Dongfengdong Street, Guangzhou, China \\ cmah05@163.com
}

\begin{abstract}
Keywords: $\mathrm{SF}_{6}$, recycling and reuse, "SF 6 Purified in Multi-channel (SFPM)" technology Abstract. As an excellent insulating medium, sulfur hexafluoride $\left(\mathrm{SF}_{6}\right)$ is widely used in electrical equipment, despite its high global warming potential. Besides, a series of hazardous decomposition products can be formed after long-term use of $\mathrm{SF}_{6}$, so it is very important for power companies to recycle $\mathrm{SF}_{6}$ in electrical equipment that is in overhaul or to be retired. To solve this problem, a "SF 6 Purified in Multi-channel (SFPM)" technology was developed, which comprises washing, adsorption, distillation, curing and exhaust gas circulation processes. The specific purification processes by which disused $\mathrm{SF}_{6}$ is purified can be customized according to its quality and the quality of $\mathrm{SF}_{6}$ after treatment meets the standards of "Industrial Sulfur Hexafluoride" (GB/T 12022-2006) and IEC 60480-2004, with zero emission of $\mathrm{SF}_{6}$ achieved during the whole process. Equipment for $\mathrm{SF}_{6}$ purification was developed and a base for $\mathrm{SF}_{6}$ recycling and reuse was established in 2010. During the past six years of operation and promotion, $93.630 \mathrm{t}$ of $\mathrm{SF}_{6} \mathrm{was}$ recycled and a total of $90.887 \mathrm{t}$ of treated $\mathrm{SF}_{6}$ was reused in electrical equipment at Guangdong Power Grid Corporation.
\end{abstract}

\section{Introduction}

Because of its unique insulating properties, high arc extinguishing properties, low toxicity and chemical stability, $\mathrm{SF}_{6}$ is widely used in electrical equipment such as Gas Insulated Switchgear (GIS), high-voltage transformers, high voltage circuit breakers and gas insulated transmission lines (GIL) ${ }^{[1]}$. According to the Intergovernmental Panel on Climate Change, $\mathrm{SF}_{6}$ is also the most potent greenhouse gas with a global warming potential of 23,900 times that of $\mathrm{CO}_{2}$ when compared over a

100-year period and a long atmospheric lifetime $(\sim 3200 \text { years })^{[2]}$. After a long-term use of $\mathrm{SF}_{6}$ in

electrical equipment, a series of hazardous decomposition byproducts can be formed due to high voltage electric field effect, electrical equipment discharge failure and other factors, such as $\mathrm{SF}_{4}$, $\mathrm{S}_{2} \mathrm{~F}_{10}, \mathrm{SOF}_{2}, \mathrm{SO}_{2} \mathrm{~F}_{2}, \mathrm{SOF}_{4}, \mathrm{~S}_{2} \mathrm{~F}_{10} \mathrm{O}, \mathrm{HF}, \mathrm{SO}_{2}$, etc ${ }^{[3]}$, which are all toxic and hazardous to human beings. If a leak or discharge may occur, workers and the people living around would pose a serious threat.

About 6,000 tons of $\mathrm{SF}_{6}$ gas is produced in China each year, more than $50 \%$ of which is used in the power industry, and the amount of $\mathrm{SF}_{6}$ to be replaced in repair process is about 700 tons ${ }^{[4]}$. $\mathrm{SF}_{6}$ gas consumption in power equipment industry is about 3,010 tons in 2008, about 4,590 tons in 2011 and about 4,890 tons in 2012, which shows a rapid growth rate. As the largest provincial power grid company in China, Guangdong Power Grid Corporation was equipped with lots of $\mathrm{SF}_{6}$ electrical equipment, the amount of $\mathrm{SF}_{6}$ in which has increased to 2300 tons and the amount of $\mathrm{SF}_{6}$ leakage has increased to about 40 tons by 2015 . It is very important for power companies to recycle $\mathrm{SF}_{6}$ in electrical equipment that is in overhaul or to be retired.

Some methods have been developed for $\mathrm{SF}_{6}$ purification, such as thermal treatment [5], membrane-based separation technique ${ }^{[6]}$, and adsorption treatment ${ }^{[7]}$, but these methods are not 
applicable to purify $\mathrm{SF}_{6}$ gas in large quantities quickly and efficiently. According to the special demand of $\mathrm{SF}_{6}$ gas treatment in Guangdong Power Grid Corporation, a "SF 6 Purified in Multi-channel (SFPM)" technology was developed, which comprises washing, adsorption, distillation, curing and exhaust gas circulation processes, which can change the purification processes and speed according to the quality of $\mathrm{SF}_{6}$ gas.

\section{Main Contents}

The biggest difficulty in $\mathrm{SF}_{6}$ gas purification is that how to detect and cleanup the impurity components, such as moisture, acidity, decomposition products and air, because these impurity components are low in mass ratio, changes in large range, and all need special treatment methods. Due to the special nature of impurity components in $\mathrm{SF}_{6}$ gas and the large amount of $\mathrm{SF}_{6}$ gas to be treated every year in Guangdong Power Grid Corporation, a new method should be developed to purify $\mathrm{SF}_{6}$ gas that is different in impurity components quickly and efficiently. It is the actual demand to recycle and reuse of $\mathrm{SF}_{6}$ in Guangdong Power Grid Corporation.

Quality standard of SF6 gas in China

The purity and components of $\mathrm{SF}_{6}$ gas are key factors for its insulation properties in electrical equipment, so the quality of $\mathrm{SF}_{6}$ gas is strictly standardized to keep the safety of power grid. In Guangdong Power Grid Corporation, "Sulfur hexafluoride for industrial use (GB/T 12022 2006)" works as $\mathrm{SF}_{6}$ gas quality standards, which proposed eight indicators, such as purity of $\mathrm{SF}_{6}$, mass fraction of air, mass fraction of $\mathrm{CF}_{4}$, humidity, mass fraction of acidity (calculated in $\mathrm{HF}$ ), hydrolysable fluorides (calculated in HF), mass fraction of mineral oil, and toxicity.

Table 1 Quality standard of SF6 gas in China

\begin{tabular}{cc}
\hline Indicators & Limits \\
\hline Purity of $\mathrm{SF}_{6}$ & $\geq 99.9 \%$ \\
Mass fraction of air & $\leq 0.04 \%$ \\
Mass fraction of $\mathrm{CF}_{4}$ & $\leq 0.04 \%$ \\
Humidity & $\leq 0.0005 \%$ \\
Mass fraction of acidity (calculated in HF) & $\leq 0.00002 \%$ \\
Hydrolysable fluorides (calculated in HF) & $\leq 0.00010 \%$ \\
Mass fraction of mineral oil & $\leq 0.0004 \%$
\end{tabular}

Toxicity

Non-toxic in biological experiments

Each indicator represents a special damage to the insulation property of $\mathrm{SF}_{6}$ gas and the electrical equipment, so the $\mathrm{SF}_{6}$ gas in any electrical equipment would be replaced to keep safe, if one or more indicator exceeds the limit in table 1.

Disused $\mathrm{SF}_{6}$ gas in Guangdong Power Grid Corporation

Disused $\mathrm{SF}_{6}$ gas was created in three ways. The first, $\mathrm{SF}_{6}$ gas of substandard quality was detected in routine test; the second, $\mathrm{SF}_{6}$ gas in faulty equipment was collected in repair process; and the last, $\mathrm{SF}_{6}$ gas in retired equipment was collected in dismantling process. Due to the large amount of $\mathrm{SF}_{6}$ gas, different equipments and variety of application environment, the disused $\mathrm{SF}_{6}$ gas in Guangdong Power Grid Corporation is really complicated from component content to component concentration.

a. $\mathrm{SF}_{6}$ gas of substandard quality

$\mathrm{SF}_{6}$ gas of substandard quality accounts for about $60 \%$ of the all disused $\mathrm{SF}_{6}$ gas in Guangdong Power Grid Corporation. The first reason of substandard quality is purity of $\mathrm{SF}_{6}$, which accounts for about $60 \%$; the second reason of substandard quality is humidity, which accounts for about $25 \%$; the third reason of substandard quality is mass fraction of air, which accounts for about $12 \%$. Component content changes with the source of the $\mathrm{SF}_{6}$ gas.

b. $\mathrm{SF}_{6}$ gas in faulty equipment

$\mathrm{SF}_{6}$ gas in faulty equipment accounts for about $35 \%$ of the all disused $\mathrm{SF}_{6}$ gas in Guangdong Power Grid Corporation. Due to some equipment accidents happen occasionally, a large amount of $\mathrm{SF}_{6}$ gas is was recovered to prevent the greenhouse effect. The $\mathrm{SF}_{6}$ gas in faulty equipment is a little special because there may be many kinds of byproducts which are difficult to be separated. 
c. $\mathrm{SF}_{6}$ gas in retired equipment

$\mathrm{SF}_{6}$ gas in retired equipment accounts for about $5 \%$ of the all disused $\mathrm{SF}_{6}$ gas in Guangdong Power Grid Corporation. Substandard quality of $\mathrm{SF}_{6}$ gas was not detected in routine test, but the component content in $\mathrm{SF}_{6}$ gas may be very complex.

The range of component concentration is analyzed in table 2, which shows the complexity of disused $\mathrm{SF}_{6}$ gas and importance of a flexible purification process.

Table 2 component concentration of disused $\mathrm{SF}_{6}$ gas

\begin{tabular}{ccc}
\hline Indicators & Range & Exceedance probability \\
\hline Purity of $\mathrm{SF}_{6}$ & $90.0 \% \sim 99.9 \%$ & $51 \%$ \\
Mass fraction of air & $0.02 \% \sim 0.80 \%$ & $20 \%$ \\
Mass fraction of $\mathrm{CF}_{4}$ & $0.01 \% \sim 0.10 \%$ & $4 \%$ \\
Humidity & $0.0002 \% \sim 0.0100 \%$ & $12 \%$ \\
Mass fraction of acidity (calculated in HF) & $0.00001 \% \sim 0.0020 \%$ & $8 \%$ \\
Hydrolysable fluorides (calculated in HF) & $0.00005 \% \sim 0.00300 \%$ & $1 \%$ \\
Mass fraction of mineral oil & $0.0002 \% \sim 0.0020 \%$ & $4 \%$ \\
\hline
\end{tabular}

$\mathrm{SF}_{6}$ Purified in Multi-channel (SFPM)

According to the component content of impurities $(0.1 \%$ to $10.0 \%)$ and chemical properties (melting point $-62{ }^{\circ} \mathrm{C}$, boiling point $-51{ }^{\circ} \mathrm{C}$ ) of $\mathrm{SF}_{6}$, a "SF 6 Purified in Multi-channel (SFPM)" technology was developed, which comprises pretreatment, washing, adsorption, distillation, curing and exhaust gas circulation processes. The specific purification processes by which disused $\mathrm{SF}_{6}$ is purified can be customized according to its quality and the quality of $\mathrm{SF}_{6}$ after treatment meets the standards of "Industrial Sulfur Hexafluoride" (GB/T 12022-2006) and IEC 60480-2004, with zero emission of $\mathrm{SF}_{6}$ achieved during the whole process.

"SF 6 Purified in Multi-channel (SFPM)" technology compose with 5 purification steps (as shown in Figure 1), changes in several ways according the quality of $\mathrm{SF}_{6}$ gas, and can give more than 5 solutions to speed up the purification process. SFPM technology purifies slightly polluted $\mathrm{SF}_{6}$ gas in short process and purifies heavily polluted $\mathrm{SF}_{6}$ gas in long process. No matter how great the variety of $\mathrm{SF}_{6}$ gas is, it would be purified in appropriate process.

Step 1: Gas Pretreatment. The gasification process of $\mathrm{SF}_{6}$ is endothermic reaction, so gas pretreatment with hot water bath is required to prevent liquid $\mathrm{SF}_{6}$ at the bottom of the cylinder curing, which would result in low recovery, low speed and gas waste. By heating the gas cylinder, the speed and recovery of $\mathrm{SF}_{6}$ gas gasification is improved.

Step 2: Alkaline Wash. The quality of pretreated gas is detected at on-line monitoring system to determine if an alkaline wash is necessary. Exceeded acid gases $\left(\mathrm{SO}_{2}, \mathrm{HF}\right)$ are removed in alkaline wash which is controlled by a PLC control system; so that the equipments and materials in follow process would not be damaged. The $\mathrm{SF}_{6}$ gas would be dried to remove water by a refrigeration dryer and then filtered by a floc filter to remove floc and solid impurities.

Step 3: Adsorption. The $\mathrm{SF}_{6}$ gas with little acid gases and $\mathrm{SF}_{6}$ gas treated by alkaline wash would be adsorbed by a series of Adsorbent to remove mineral oil, moisture, decomposition products, and toxic low fluoride. Considering the price and performance of catalysis, inexpensive and easily replaceable silica and activated alumina are placed upstream of the gas passage to remove impurities roughly, then A-type zeolite is placed in the medium to adsorb moisture specially, and KDHF-03 type zeolite is placed at last to as an advanced treatment of decomposition products and toxic low fluoride. The quality of $\mathrm{SF}_{6}$ would be judged after adsorption if subsequent treatment or re-adsorption is necessary. About $30 \%$ of disused $\mathrm{SF}_{6}$ gas can be purified to meet the gas quality requirements and would be separated directly. 


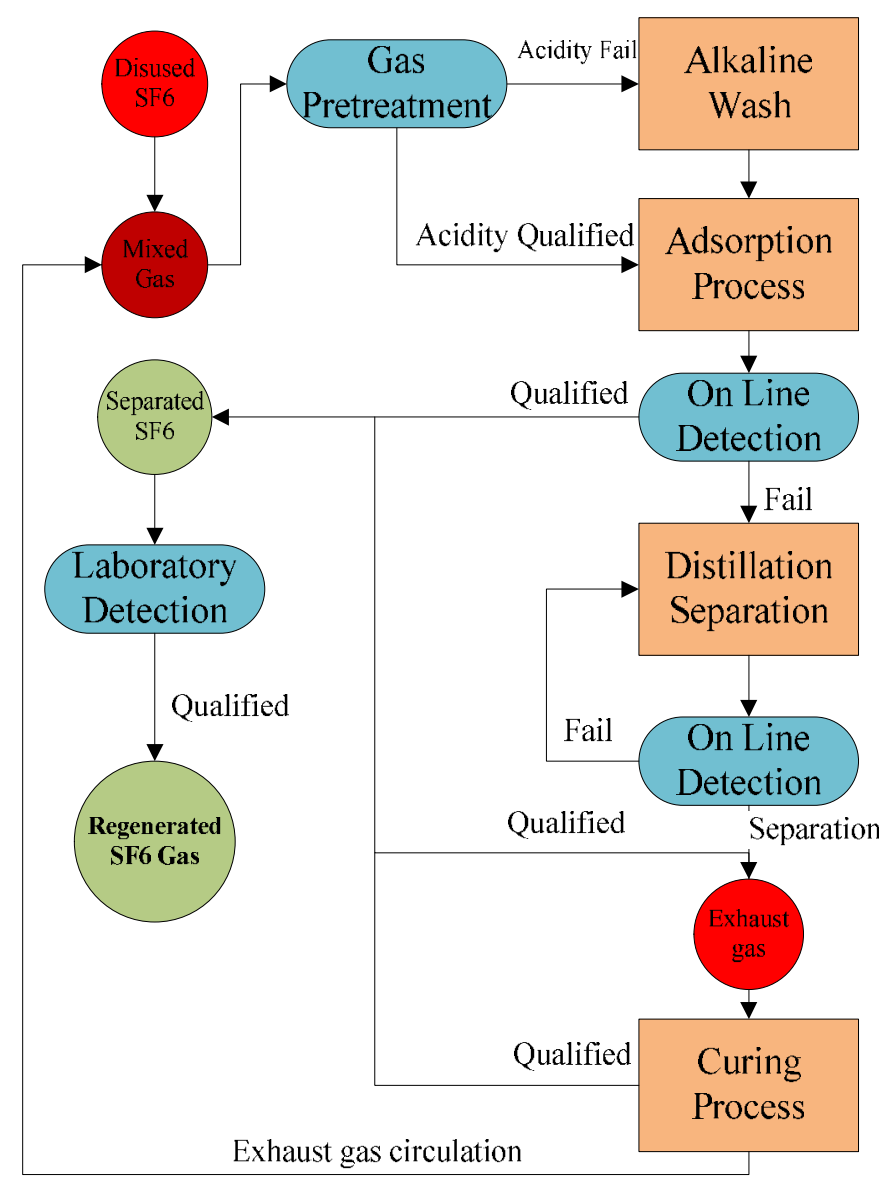

Figure. $1 \mathrm{SF}_{6}$ Purified in Multi-channel (SFPM)

Step 4: Distillation Separation. Air and remaining impurities are separated at this process quickly and efficiently. A distillation column with the processing speed of $50 \mathrm{Kg} / \mathrm{h}$ is designed under the thermodynamic theoretical calculations, which constitute the main part of SFPM technology. The unqualified $\mathrm{SF}_{6}$ gas is liquefied by a cold trap and then inputted into distillation column. The gaseous air and other remaining impurities would be separated easily because there is a great relative volatility between gaseous air and liquefied $\mathrm{SF}_{6}$. Separated $\mathrm{SF}_{6}$ is transferred to a special container, and the light component with a small amount of $\mathrm{SF}_{6}$.is transferred to an exhaust gas storage tank. If the processing efficiency is not satisfied, a re-distillation separation would be carried. About $60 \%$ of disused $\mathrm{SF}_{6}$ gas can be purified to meet the gas quality requirements at this process.

Step 5: Curing process. Zero Emission of $\mathrm{SF}_{6}$ is achieved at this step by exhaust gas treatment and recycling. The exhaust gas compressor is automatically started when the storage tank reaches a set pressure, and a cold trap under low mode would cure trace $\mathrm{SF}_{6}$.in exhaust gas. Cured $\mathrm{SF}_{6}$.is gasified and separated as clean gas. The exhaust gas would be draw out by a vacuum pump, filtered by an exhaust gas filter device, neutralized by an alkaline solution pool, mixed with untreated $\mathrm{SF}_{6}$.and retreated. About $10 \%$ of disused $\mathrm{SF}_{6}$ gas can be purified to meet the gas quality requirements at this process.

$\mathrm{SF}_{6}$ Purification Equipment

A highly integrated purification equipment(as shown in Figure 2) was develop in 2010 based on $\mathrm{SF}_{6}$ Purified in Multi-channel (SFPM) technology, which is highly automated, can be control remotely with communications technology, purify disused $\mathrm{SF}_{6}$ gas quickly and efficiently, and can regenerate gas to meet the quality standards of $\mathrm{SF}_{6}$ gas. The purification equipment is controlled by a PLC system, equipped with $\mathrm{SF}_{6}$ gas analysis instrumentation and air quality monitoring instrumentation for on line detection, and connected with chromatography instruments in laboratory by a pipe system. 
$\mathrm{SF}_{6}$ purification equipment was made in modular combinations, which means that each module is independent and can union by connecting structure. This set of equipment is divided into three major functional unit area (A, B, C zone), that are installed in guide rail and can move in direction of the specified. It is very convenient for purification equipment to be operated, maintained and repaired, because each functional area is equipped with a deployable wingspan mask.

Other characteristics of $\mathrm{SF}_{6}$ purification equipment: 1) equipped with digital weighing system, 2) zeolite being regenerated automatically, 3) equipped with remote automation operating system, 4) secondary treatment can be carried in cycle purification process, 5) zero emission of $\mathrm{SF}_{6}$ achieved.
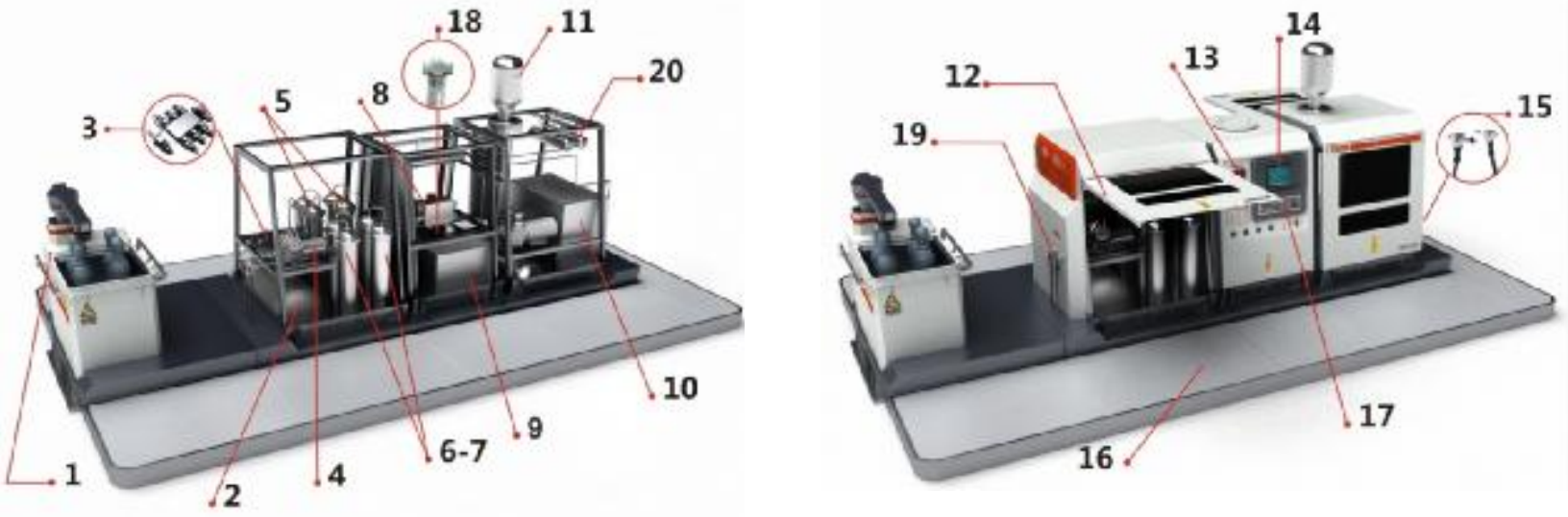

Figure. $2 \mathrm{SF}_{6}$ Purification Equipment

(1-Cylinder Pretreatment System, 2-Buffer Tank, 3-Solenoid Valve, 4-Vacuum Compressor, 5-A

Series of Zeolite, 6-7-Alkaline Wash Tank, 8-Vacuum Pump, 9-Refrigeration Dryer, 10-Cold Trap, 11-Distillation Column, 12-Wingspan Style Cover, 13-Environmental Monitoring System, 14-PLC Control System, 15-Nozzles, 16-Operator Station, 17-On Line Detecting Instrumentation, 18-SF 6 Compressor, 19-Self-styled Inlet Connector, 20-exhaust gas tank)

\section{Results}

Treatment effect of SFPM technology

In order to verify the purification efficiency of $\mathrm{SF}_{6}$ Purified in Multi-channel (SFPM) technology and $\mathrm{SF}_{6}$ purification equipment, $5 \mathrm{SF}_{6}$ gas samples was collected from 5 different transformer substations in 2010, which were named Sample A, Sample B, Sample C, Sample D and Sample E. The quality of each sample is really different and complex (as shown in Table 2), but each sample was regenerated to meet quality standard of "Sulfur hexafluoride for industrial use (GB/T 12022 2006)", which means that these regenerated $\mathrm{SF}_{6}$ could be reused to electrical equipment as insulating medium.

1) Purification efficiency of impurities

The purification efficiency of impurities by $\mathrm{SF}_{6}$ Purified in Multi-channel (SFPM) technology and $\mathrm{SF}_{6}$ purification equipment is shown in Table 2, which indicate that the 7 indicators set by "Sulfur hexafluoride for industrial use (GB/T 12022 2006)" is qualified after treatment, whatever the concentration of impurities changes drastically. 
Table 2 Purification efficiency of impurities

\begin{tabular}{ccccccccc}
\hline \multirow{2}{*}{ Samples } & Treatment & $\begin{array}{c}\mathrm{H}_{2} \mathrm{O} \\
{\left[10^{-6}\right]}\end{array}$ & $\begin{array}{c}\text { AIR } \\
{[\%]}\end{array}$ & $\mathrm{CF}_{4}[\%]$ & $\begin{array}{c}\text { Hydrolysable } \\
\text { fluorides } \\
{\left[10^{-6}\right]}\end{array}$ & $\begin{array}{c}\text { Acidity } \\
{\left[10^{-6}\right]}\end{array}$ & $\begin{array}{c}\text { Mineral } \\
\text { Oil }\left[10^{-6}\right]\end{array}$ & $\begin{array}{c}\text { Purity } \\
{[\%]}\end{array}$ \\
\hline \multirow{2}{*}{ A } & Before & 15.0 & 0.081 & 0.003 & 0.453 & 0.032 & 2.1 & 98.89 \\
& After & 2.3 & 0.010 & 0.002 & 0.032 & 0.011 & 0.2 & 99.99 \\
& Before & 13.0 & 0.006 & 0.008 & 0.684 & 0.309 & 5.7 & 99.01 \\
B & After & 1.4 & 0.005 & 0.002 & 0.083 & 0.043 & 0.3 & 99.99 \\
& Before & 12.5 & 0.072 & 0.004 & 0.365 & 0.412 & 6.1 & 99.50 \\
C & After & 1.6 & 0.008 & 0.001 & 0.032 & 0.008 & 0.5 & 99.99 \\
& Before & 10.9 & 0.056 & 0.043 & 0.451 & 0.523 & 8.2 & 98.67 \\
& After & 1.3 & 0.009 & 0.006 & 0.026 & 0.031 & 0.6 & 99.99 \\
& Before & 12.8 & 0.022 & 0.032 & 0.356 & 0.432 & 6.3 & 99.53 \\
E & After & 1.4 & 0.010 & 0.004 & 0.052 & 0.076 & 0.8 & 99.99 \\
& Standard & $\leq 5.0$ & $\leq 0.040$ & $\leq 0.040$ & $\leq 1.000$ & $\leq 0.200$ & $\leq 4.0$ & $\geq 99.9$ \\
\hline
\end{tabular}

2) Purification efficiency of subfluoride

It is difficult to remove subfluoride in $\mathrm{SF}_{6}$ gas because subfluoride is similar to $\mathrm{SF}_{6}$ in nature. As shown in Table 3, subfluoride in Sample A, Sample B and Sample C were removed thoroughly, which means that the purification efficiency of subfluoride by $\mathrm{SF}_{6}$ purification equipment is satisfied.

Table 3 Purification efficiency of subfluoride

\begin{tabular}{ccccc}
\hline Sample & Treatment & $\mathrm{SOF}_{2} / \%$ & $\mathrm{~S}_{2} \mathrm{OF}_{10} / \%$ & $\mathrm{SOF}_{4} / \%$ \\
\hline \multirow{2}{*}{$\mathrm{A}$} & Before & Undetected & 0.002 & 0.004 \\
& After & Undetected & Undetected & Undetected \\
\multirow{2}{*}{$\mathrm{B}$} & Before & 0.021 & 0.032 & Undetected \\
& After & Undetected & Undetected & Undetected \\
\multirow{2}{*}{$\mathrm{D}$} & Before & 0.011 & Undetected & 0.002 \\
& After & Undetected & Undetected & Undetected \\
\hline
\end{tabular}

3) Purification efficiency of toxicity

The purification efficiency of toxicity was tested by animal experiment. There fed mice were put in confined space that was filled with $79 \%$ regenerated $\mathrm{SF}_{6}$ and $21 \%$ oxygen. No sign of toxicity in mice was found after 48 hours, which indicate that the purification efficiency of toxicity is satisfied.

4) Insulation property of regenerated $\mathrm{SF}_{6}$

The regenerated $\mathrm{SF}_{6}$ gas was filled into a $150 \mathrm{kV}$ GIS equipment that is applied $150 \mathrm{kV}$ voltage in one electric Laboratory of Guangdong Power Grid Corporation. None partial discharge phenomenon or breakdown phenomenon was found, which indicate that the insulation property of toxicity is satisfied.

3.2 Recycling and reuse of $\mathrm{SF}_{6}$ gas in Guangdong Power Grid Corporation

A highly automated Recycling and reuse basement for $\mathrm{SF}_{6}$ gas was established in 2010 to meet the demand of $\mathrm{SF}_{6}$ gas emission reduction in Guangdong Power Grid Corporation. A life-cycle management concept of $\mathrm{SF}_{6}$ gas was carried during the process of $\mathrm{SF}_{6}$ gas emission reduction to achieve a "decentralized recycling, centralized purification and controlled reuse" model. Disused $\mathrm{SF}_{6}$ gas would be sent to this basement and reused to electric device after purification and testing qualified. 
The amount of recycled $\mathrm{SF}_{6}$ gas comes up to 93.630 tons (as shown in figure 3 ) from February 2010 to December 2015, which is equivalent to reduce 2.23 million tons of carbon dioxide emissions, and the amount of reused $\mathrm{SF}_{6}$ gas comes up to 90.887 tons, which reduced the gas purchase cost by 12.72 million Yuan.

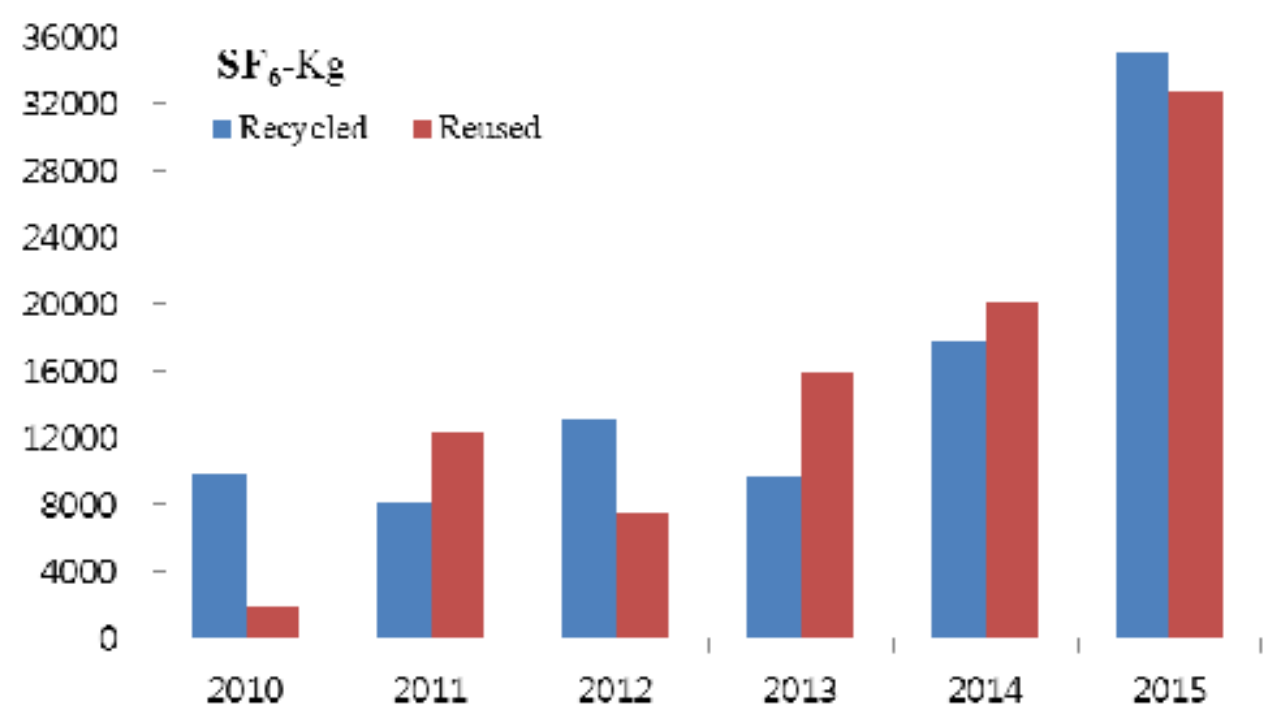

Figure. 3 Recycling and reuse of $\mathrm{SF}_{6}$ gas in Guangdong Power Grid Corporation

\section{Conclusion}

The quality standard of $\mathrm{SF}_{6}$ gas is very strict and a stricter standard is to be promulgated officially, so it is a difficult to treat large amount of $\mathrm{SF}_{6}$ gas efficiently. The $\mathrm{SF}_{6}$ Purified in Multi-channel (SFPM) technology that consist of five purify process can remove impurities, subfluoride and toxicity in disused SF6 gas, and regenerate disused SF6 gas to meet the requirements in reuse. A new kind of $\mathrm{SF}_{6}$ purification equipment was developed, a basement for Recycling and reuse of $\mathrm{SF}_{6}$ gas in Guangdong Power Grid Corporation and a great achievement was created.

The successful experience of Guangdong Power Grid Corporation shows that recycling and reuse of $\mathrm{SF}_{6}$ is technically sound, economically viable, beneficial to society and the environment, and thus important for building a green power grid and a green society.

\section{References}

[1] M. Maiss, C.A.M. Brenninkmeijer, Atmospheric SF6: trends, sources and prospects, Environ. Sci. Technol. 32 (1998), 3077-3086

[2] V. Mohindra, H. Chase, H.H. Sawin, M.T. Mocella, Abatement of perfluorocompounds (PFCS) in a microwave tubular reactor using $\mathrm{O}_{2}$ as an additive gas, IEEE Trans. Plasma Sci. 10 (1997), 399-407

[3] Wang $\mathrm{Yu}$, Differences of $\mathrm{SF}_{6}$ byproducts in simulated electric equipment of overheating faults in different condition, 1st International Conference on Energy and Environmental Protection, $\operatorname{ICEEP}(2012)$

[4] Wang Yu, Li Li, Tang Longhua, et al. Calculation on Electric Field Distribution of Typical 500 kV Current Transformer Based on ANSYS Analysis, Guangdong Electric Power(2014), 73-78

[5] M. Freemantle, Membranes for gas separation, Chem. Eng. News. 83(2005), 49-57

[6] Soonjae Lee, JongSukLee, Minwoo Lee, et al. Separation of sulfur hexafluoride ( $\mathrm{SF}_{6}$ ) from ternary gas mixtures using commercial polysulfone (PSf) hollow fiber membranes, Journal of 
Membrane Science. 452(2014), 311-318

[7] Yu-Chun Chiang, Po-Yun Wu. Adsorption equilibrium of sulfur hexafluoride on multi-walled carbon nanotubes, Journal of Hazardous Materials. 178(2010), 729-738 